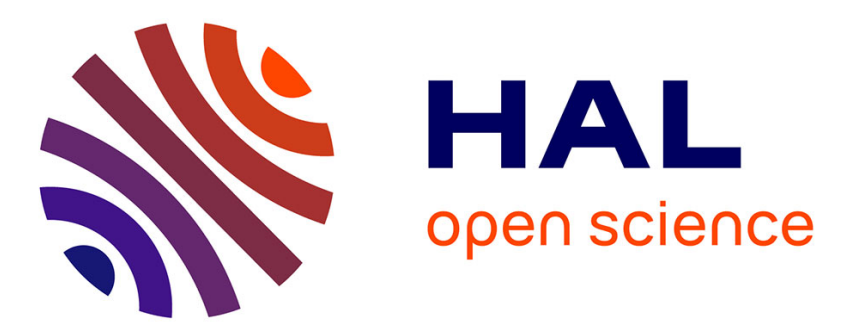

\title{
The Scaled SLW model of gas radiation in non-uniform media based on Planck-weighted moments of gas absorption cross-section
}

\author{
Vladimir Solovjov, Frédéric André, Brent Webb, Denis Lemonnier
}

\section{To cite this version:}

Vladimir Solovjov, Frédéric André, Brent Webb, Denis Lemonnier. The Scaled SLW model of gas radiation in non-uniform media based on Planck-weighted moments of gas absorption crosssection. Journal of Quantitative Spectroscopy and Radiative Transfer, 2018, 206, pp.198-212. 10.1016/j.jqsrt.2017.11.012 . hal-01934760

\author{
HAL Id: hal-01934760 \\ https://hal.science/hal-01934760
}

Submitted on 23 Apr 2019

HAL is a multi-disciplinary open access archive for the deposit and dissemination of scientific research documents, whether they are published or not. The documents may come from teaching and research institutions in France or abroad, or from public or private research centers.
L'archive ouverte pluridisciplinaire HAL, est destinée au dépôt et à la diffusion de documents scientifiques de niveau recherche, publiés ou non, émanant des établissements d'enseignement et de recherche français ou étrangers, des laboratoires publics ou privés. 


\title{
The Scaled SLW Model of Gas Radiation in Non-uniform Media Based on Planck-weighted Moments of Gas Absorption Cross-section
}

\author{
Vladimir P. Solovjov*\$, Frederic Andre ${ }^{* *}$, Denis Lemonnier ${ }^{* * *}$, Brent W. Webb \\ *Dept. of Mech. Engineering, 435 CTB, Brigham Young University, Provo, UT 84602, USA \\ ${ }^{* *}$ Centre de Thermique et d'Energétique de Lyon, INSA de Lyon, 9 rue de la Physique, 69621 \\ Villeurbanne, France \\ *** ISAE-ENSMA, BP 40109, 86961 Futuroscope Chasseneuil Cedex, France \\ ${ }^{\S}$ Corresponding author: Email: lemberg.v@gmail.com
}

\begin{abstract}
The Scaled SLW model for prediction of radiation transfer in non-uniform gaseous media is presented. The paper considers a new approach for construction of a Scaled SLW model. In order to maintain the SLW method as a simple and computationally efficient engineering method special attention is paid to explicit non-iterative methods of calculation of the scaling coefficient. The moments of gas absorption cross-section weighted by the Planck blackbody emissive power (in particular, the first moment - Planck mean, and first inverse moment - Rosseland mean) are used as the total characteristics of the absorption spectrum to be preserved by scaling. Generalized SLW modelling using these moments including both discrete gray gases and the continuous formulation is presented. Application of line-by-line look-up table for corresponding ALBDF and inverse ALBDF distribution functions (such that no solution of implicit equations is needed) ensures that the method is flexible and efficient. Predictions for radiative transfer using the Scaled SLW model are compared to line-by-line benchmark solutions, and predictions using the Rank Correlated SLW model and SLW Reference Approach. Conclusions and recommendations regarding application of the Scaled SLW model are made.
\end{abstract}

Keywords: gas radiation, non-uniform, SLW, Rank Correlated SLW Model, Reference Approach SLW, Scaled SLW, moments of absorption cross-section 


\section{NOMENCLATURE}

a gray gas weights

$C$ gas absorption cross-section $\left[\mathrm{m}^{2} / \mathrm{mol}\right]$

$E \quad$ radiation emissive power $\left[\mathrm{W} / \mathrm{m}^{2}\right]$

$F \quad$ ALBDF

$I \quad$ radiation intensity $\left[\mathrm{W} / \mathrm{m}^{2} / \mathrm{sr}\right]$

$L \quad$ gas layer thickness [m]

$\ell \quad \ell$-distribution

$N \quad$ gas molar density $\left[\mathrm{mol} / \mathrm{m}^{3}\right]$

$p \quad$ gas total pressure [atm]

$s \quad$ path variable [m]

$T \quad$ gas temperature $[\mathrm{K}]$

$u \quad$ scaling coefficient

$Y \quad$ gas specie mole fraction

\section{Greek symbols}

$\beta \quad \ell$-distribution overlapping parameter

$\phi \quad$ symbolic notation for given gas thermodynamic state, $\phi=\{T, Y, p\}$

$\xi \quad$ continuous variable as a limit of a discrete absorption cross-section

$\kappa \quad$ gas absorption coefficient $\left[\mathrm{m}^{-1}\right]$

$\mu \quad$ moment

$\eta \quad$ wavenumber $\left[\mathrm{cm}^{-1}\right]$

$\sigma \quad$ Stefan-Boltzmann constant, $5.67 \times 10^{-8} \mathrm{~W} / \mathrm{m}^{2} / \mathrm{K}^{4}$

$\tau \quad$ transmissivity

\section{Subscripts}

$b \quad$ blackbody

$j \quad$ gray gas number

$n \quad$ number of gray gases in the model

$P \quad$ Planck mean

$R \quad$ Rosseland mean

$s \quad$ order of the moment

loc local

ref reference 


\section{INTRODUCTION}

Among the global methods of gas radiation, the Spectral-Line-Weighted-sum-of-gray-gases (SLW) model has been demonstrated to be a simple, accurate, and efficient method of spectral modelling of radiation transfer in gaseous media [1]. A primary challenge of global methods is spectral modelling of radiation transfer in non-uniform media (non-isothermal and/or nonhomogeneous scenarios), where the gas absorption spectrum changes with spatial location due to changes in local thermodynamic state. Approaches based on the assumption of correlated gas absorption spectra have been developed [1-6]. Greater accuracy in these approaches comes generally at the expense of greater sophistication, more complexity, greater difficulty in implementation, and higher associated computational cost. This work concerns itself with the scaled spectrum assumption in the context of the Spectral Line Weighted-sum-of-gray-gases (SLW) model.

The SLW method considered in this work is an extension and further development of the global WSGG method originally proposed by Song and Viskanta [4]. They recognized the difficulty which arises in WSGG modelling of gas radiation in non-uniform media. They noted that spectral integration of monochromatic RTE over the gray gas wavenumber intervals yields appearance of numerous so-called Leibnitz terms. These terms arise in the integration because the boundaries of the gray gas wavenumber intervals vary with spatial location in non-uniform media if the supplemental cross-sections defining the gray gas wavenumber intervals are fixed [2-6]. Simple neglect of these additional terms can yield significant error in the prediction of radiative transfer. It was proposed by Song and Viskanta that the gray gas spectral intervals be fixed to avoid the appearance of the Leibnitz terms by varying the supplemental absorption cross-sections in such a way that the spectral intervals obtained at some chosen reference thermodynamic state are maintained the same at all local states in the medium. However, the approach was not implemented in the original paper, nor in the following applications of the WSGG method [7]. Despite this issue, the WSGG method continues to be attractive to researchers, and further improvement of the method has been developed recently [8-12]. The different versions of the WSGG method are distinguished mainly by the difference in mathematical representations of the gray gas absorption coefficients and the corresponding weights. For example, recent work has proposed the determination of coefficients in the WSGG model through the $i$ ) superposition of correlations for a mixture of gas species and soot, and $i$ ) superposition of the separate coefficients for each specie (and soot) [9]. These correlations are obtained from the high resolution spectral database HITEMP-2010 [13] by fitting emittance data

for a range of thermodynamic conditions. The examples considered in $[11,12]$ demonstrate satisfactory accuracy for non-isothermal, non-homogeneous cases.

The classical WSGG method does not possess the flexibility of the SLW method which, unlike the WSGG method, can be used with an arbitrary number of gray gases and any gas 
compositions. However, although the WSGG method may not be sufficiently accurate to provide benchmark results, its robustness, consistency and computational efficiency make it a viable alternative to more sophisticated models, especially in applications in which thermal radiation is only one part of several other complex, coupled modeled phenomena.

Approaches in the SLW methods are based on assumptions of correlated/scaled absorption spectra which can be reduced to the assumption on which separation of dependence of gas absorption coefficient on space variable (and therefore, on the local gas thermodynamic state) and on wavenumber. The usual approaches are based on specification of a reference thermodynamic state and construction of the associated SLW reference histogram absorption spectrum at this state. The local histogram spectrum at other spatial locations is then scaled to the reference spectrum by a scaling coefficient which can be different for different gray gases (correlated models), or by a single coefficient (scaled models). Because this assumption, in general, is not valid for real spectra, accuracy of the predictions depends critically on the choice of reference state. The reference gas temperature $T_{r e f}$ has been chosen from some physical consideration which has included the maximum temperature in the system, the minimum temperature in the system, the volume-averaged temperature, the Planck mean temperature, and the emission-weighted temperature $[6,14]$.

Application of the correlated spectrum assumption does not necessarily provide optimal accuracy of prediction of radiative quantities. For systems with large temperature and/or concentration gradients, the dependence of the spectral properties on these properties can produce significant errors. One way to solve this temperature dependence problem is to model the absorption coefficient's dependence on the temperature as was done in the Multi-Group Full Spectrum $k$ distribution Method (MGFSK) [15]. The MGFSK Method provides good results for inhomogeneous media, and the accuracy may be increased by increasing the number of groups. However, the method remains approximate. A more accurate but more computationally expensive alternative is presented by Tencer and Howell [14]. Their multi-source full spectrum $k$-distribution method is capable of providing exact results for one-dimensional geometries with piecewise constant temperature and absorption coefficient in the same way that the full spectrum $k$-distribution method is able to provide exact results for homogeneous media. The approach as outlined in [14] provides a significant increase in accuracy when compared to other $k$ distribution based approaches while remaining significantly less computationally expensive than a standard line-by-line solution. The errors introduced by this method are entirely due to the multilayer approximation. As a result, the method converges linearly as the number of layers is increased. This allows the error relative to the line-by-line solution to be estimated.

The need for simple, accurate engineering approaches for the prediction of radiation transfer in non-uniform high temperature gas applications remains, particularly in comprehensive reacting flow modeling applications. Recent progress in SLW modelling, including the development of 
the Generalized SLW model [2] and the Rank Correlated SLW model [3, 16], opens new opportunities for a model developed independently on the assumption of a scaled spectrum. The scaled spectrum assumption has never been explored in the framework of the SLW model, but it has potential to improve performance of the SLW method when correlated models fail. The scaled SLW models which are outlined here are not intended to replace the correlated SLW models developed earlier, but rather to complement the correlated SLW model.

In the scaled spectral model it is assumed that the absorption cross-sections of a particular gas or gas mixture at two different local thermodynamic states denoted symbolically as $\phi_{1}=\left(T_{1}, Y_{1}, p\right)$ and $\phi_{2}=\left(T_{2}, Y_{2}, p\right)$ are related by a simple scaling for the entire spectrum (for all wavenumbers $\eta>0)$ as

$C_{\eta}\left(\phi_{2}\right)=u\left(\phi_{1}, \phi_{2}\right) C_{\eta}\left(\phi_{1}\right)$

The scaled spectrum assumption is a particular and more restrictive case of the correlated spectrum assumption. However, application of the scaled model may have some advantages. Correlated spectrum models rely on how well real spectra are correlated but they cannot overcome the fact that real spectra are never completely correlated. Consequently, in the case of highly non-uniform media the prediction of radiative transfer can be inaccurate. Scaled spectral models may improve performance with the help of a more informed choice of scaling coefficient $u\left(\phi_{1}, \phi_{2}\right)$ for better representation of gas absorption spectra at the local thermodynamic state in the prediction of radiative transfer in non-uniform media. Further, the scaled model may have the advantage of being simpler in its construction and implementation.

Once the scaling coefficient is determined, the scaled SLW model can be readily constructed. Therefore, the critical element of the scaled model is the development of an efficient method of calculating the scaling coefficient $u\left(\phi_{1}, \phi_{2}\right)$. A global scaled model requires the scaling coefficient for the entire (full) spectrum. However, for particular radiation problems not all spectral regions contribute equally to the total radiation energy transfer. Emphasizing more important wavenumber regions by a properly chosen weighting function and requiring the scaling coefficient to preserve total characteristics of the absorption coefficient at the local state can make scaling of some chosen reference spectrum to a spectrum at a local state more efficient and more accurate.

The works of Godson [as summarized in 17] and Weinreb and Neuendorfer [18] appear to be the first to investigate scaled spectrum modeling. Modest subsequently proposed the scaled FSK model, demonstrating some advantage in accuracy of radiative transfer predictions over correlated FSK models [19]. In that approach computation of the local scaling coefficient was performed by solution of the implicit integral equation 


$$
\int_{0}^{\infty} I_{b \eta}\left(T_{0}\right) e^{-\kappa_{\eta}(\eta, \phi) L_{m}} d \eta=\int_{0}^{\infty} I_{b \eta}\left(T_{0}\right) e^{-\kappa_{\eta}\left(\eta, \phi_{0}\right) u\left(\phi, \phi_{0}\right) L_{m}} d \eta
$$

The relationship of Eq. (1) provides the correct evaluation of radiation transmitted through a plane layer of thickness $L_{m}$, which is the mean beam length. Following the FSK model formulation, this equation may be expressed in terms of $k$-distributions by

$$
\int_{0}^{1} e^{-\kappa^{*}\left(T_{0}, \phi, g\right) L_{m}} d g=\int_{0}^{1} e^{-\kappa^{*}\left(T_{0}, \phi_{0}, g_{0}\right) u\left(\phi, \phi_{0}\right) L_{m}} d g_{0}
$$

Equation (2) is an implicit integral equation in the local scaling coefficient $u\left(\phi, \phi_{0}\right)$, and is solved iteratively to find the coefficient. It was noted by Modest in [20] that a simplified scaling function would be needed without the use of an implicit equation to make the scaled model more efficient and attractive for adoption.

This paper considers different approaches for construction of the Scaled SLW model. In order to maintain the SLW method as a simple and computationally efficient engineering method special attention is paid to explicit, non-iterative methods of calculation of the scaling coefficient. The moments of gas absorption cross-section weighted by the Planck blackbody emissive power (in particular, the first moment - Planck mean, and the first inverse moment - Rosseland mean) are used as the total characteristics of the absorption spectrum to be preserved by scaling. Generalized SLW modelling of these moments (including both discrete gray gases and the continuous formulation) and the LBL look-up table for corresponding distribution functions (ALBDF and inverse ALBDF, such that no solution of implicit equations is needed) ensures that the method is flexible and efficient.

The scaling assumption presented in this paper is not new. It is similar to assumptions previously used in narrow band and global methods. The objective of this work is to develop the scaled model within the SLW model framework which is simple yet accurate enough for engineering applications. Scaled SLW model predictions are compared to LBL benchmark solutions, to the most recent advancement in correlated SLW modeling (Rank Correlated SLW model), and to the traditional SLW model reference approach. Conclusions and recommendations on application of the Scaled SLW model are made.

\section{THE SCALED SLW MODEL}

\section{Scaled gas absorption spectra assumption}

Consider a gas characterized by the spectral absorption cross-section $C_{\eta}(\phi)$ defined at the gas thermodynamic state symbolically denoted by a set of properties $\phi=\{T, Y, p\}$, where $T$ is the gas temperature, $Y$ is the gas mole fraction, and $p$ is the total pressure. As illustrated in Fig. 1, 
the gas absorption cross-section $C_{\eta}(\phi)$ is said to be scaled if the entire spectra $C_{\eta}\left(\phi_{1}\right)$ and $C_{\eta}\left(\phi_{2}\right)$ at any two different thermodynamic states $\phi_{1}$ and $\phi_{2}$ for all $\eta>0$ are related by the simple equation

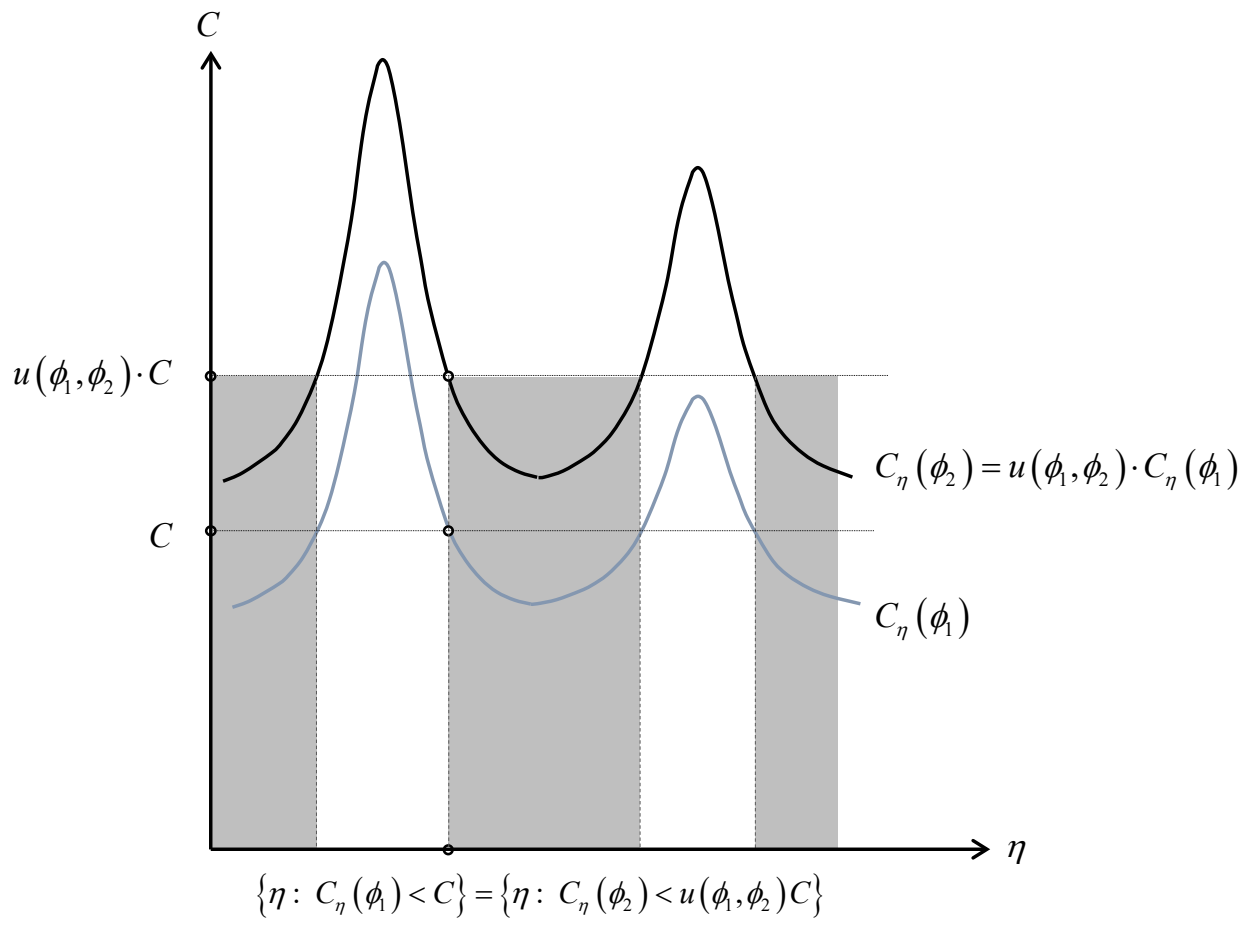

Figure 1. Scaled gas absorption cross-sections.

$C_{\eta}\left(\phi_{2}\right)=u\left(\phi_{1}, \phi_{2}\right) C_{\eta}\left(\phi_{1}\right)$

Here, the scaling coefficient $u\left(\phi_{1}, \phi_{2}\right)$ depends only on the two thermodynamic states $\phi_{1}$ and $\phi_{2}$, and is independent of wavenumber $\eta$. The assumption of scaled spectrum preserves the wavenumber intervals, with the implication being that the wavenumber intervals for which absorption cross-section at State 1 is less than any prescribed value $C$ is the same as the set of wavenumbers for which the absorption cross-section at State 2 is less than the scaled value $u\left(\phi_{1}, \phi_{2}\right) C$. This is stated mathematically as

$\left\{\eta: C_{\eta}\left(\phi_{1}\right)<C\right\}=\left\{\eta: C_{\eta}\left(\phi_{2}\right)<u\left(\phi_{1}, \phi_{2}\right) C\right\}$

Therefore, the assumption of scaled spectrum is a particular case of the more general assumption of rank correlated spectrum $[3,16]$. 
If one particular thermodynamic state is fixed as a reference state $\phi_{\text {ref }}$, then the absorption crosssection at any local state $C_{\eta}\left(\phi_{l o c}\right)$ can be found by scaling the reference absorption cross-section $C_{\eta}\left(\phi_{\text {ref }}\right)$ as shown in Eq. (5)

$$
C_{\eta}\left(\phi_{l o c}\right)=u\left(\phi_{l o c}, \phi_{r e f}\right) C_{\eta}\left(\phi_{r e f}\right)=u\left(\phi_{l o c}\right) C_{\eta}\left(\phi_{r e f}\right)=u_{l o c} C_{\eta}\left(\phi_{r e f}\right)
$$

The objective of the Scaled SLW model is to find the local scaling coefficient $u_{l o c}$ which provides the best possible solution for radiative transfer in non-uniform media. Further, optimization of the local scaling coefficient should not be too complicated. For engineering applications it may be preferable to settle for somewhat less accurate solutions in favor of the computational economy offered by an explicit formula for calculation of the scaling coefficient. The objective of the present paper is to find a scaling coefficient which preserves some important global physical characterizations such as the moments of the absorption cross-section.

\section{Moments of gas absorption cross-section}

Moments are important total characterizations of the spectral dependence of the gas absorption cross-section. In general, the spectral absorption cross-section can be reconstructed from its moments. Preservation of moments by the scaling coefficient can be a criterion for its construction.

The $s^{\text {th }}$ moment $\mu_{s}\left(\phi, T_{b}\right)$ of the gas absorption cross-section $C_{\eta}(\phi)$ at thermodynamic state $\phi$ weighted by the Planck spectral blackbody emissive power $E_{b \eta}\left(T_{b}\right)$ at the blackbody temperature $T_{b}$ can be defined generally as

$$
\mu_{s}\left(\phi, T_{b}\right)=\int_{0}^{\infty}\left[C_{\eta}(\phi)\right]^{s} \frac{E_{b \eta}\left(T_{b}\right)}{E_{b}\left(T_{b}\right)} d \eta
$$

where the moment order $s$ can be any non-zero real number, and $E_{b}\left(T_{b}\right)=\sigma T_{b}^{4}$ is the total blackbody emissive power at temperature $T_{b}$. Combining Eqs. (5) and (6), it can be shown that the moments $\mu_{s}\left(\phi_{1}, T_{b}\right)$ and $\mu_{s}\left(\phi_{2}, T_{b}\right)$ of the scaled gas absorption cross-sections $C_{\eta}\left(\phi_{1}\right)$ and $C_{\eta}\left(\phi_{2}\right)$ at any two different thermodynamic states $\phi_{1}$ and $\phi_{2}$ are related by the equation

$$
\mu_{s}\left(\phi_{1}, T_{b}\right)=\left[u\left(\phi_{1}, \phi_{2}\right)\right]^{s} \mu_{s}\left(\phi_{2}, T_{b}\right)
$$

This relationship for the case of a fixed reference state as in Eq. (5) can be rewritten as

$$
\mu_{s}\left(\phi_{l o c}, T_{b}\right)=u_{l o c}^{s} \mu_{s}\left(\phi_{r e f}, T_{b}\right)
$$

Then the local scaling coefficient $u_{l o c}$ can be found explicitly by

$$
u_{l o c}=\left[\frac{\mu_{s}\left(\phi_{l o c}, T_{b}\right)}{\mu_{s}\left(\phi_{r e f}, T_{b}\right)}\right]^{1 / s}
$$


The significance of Eq. (9) is that the scaling coefficient may be found explicitly, rather than through the solution of an implicit equation. This equation will be applied for calculation of the local scaling coefficient of absorption cross-section. The scaled model based on moments assumes a perfect statistical independence between some reference spectrum and the spectral scaling coefficient constructed by dividing any other spectrum by the reference. This assumption is used implicitly to derive Eq. (7).

In this work the focus will be on two important particular moments: the $1^{\text {st }}$ moment $(s=1)$ and the $1^{\text {st }}$ inverse moment $(s=-1)$ of the gas absorption cross-section.

\section{SLW modelling of the moments of gas absorption cross-section}

The SLW method is based on application of the absorption line blackbody distribution function (ALBDF) defined as [1]:

$F\left(C, \phi, T_{b}\right)=\int_{0}^{\infty} H\left[C_{\eta}(\phi)-C\right] \frac{E_{b \eta}\left(T_{b}\right)}{E_{b}\left(T_{b}\right)} d \eta$

where $C$ is the absorption cross-section and $H$ is the Heaviside unit-step function. Its value represents the fraction of the total emissive power $E_{b}\left(T_{b}\right)$ at blackbody temperature $T_{b}$ which lies in the portions of the spectrum where the gas absorption cross-section $C_{\eta}(\phi)$ is below the prescribed value $C$.

Following the classical SLW model, discretization of the gray gases is performed with the help of discrete values of the supplemental cross-section $\tilde{C}_{j}, j=0,1, \ldots, n$, where $n$ is the number of gray gases in the model. The gray gas is defined by its absorption cross-section $C_{j}$, usually chosen as the geometric mean of adjacent supplemental cross-sections. As is shown in ref. [2], the SLW method models the moments as follows:

$1^{\text {st }}$ moment ("Planck mean" of the absorption cross-section):

$C_{P}\left(\phi, T_{b}\right)=\mu_{1}\left(\phi, T_{b}\right)=\int_{0}^{\infty} C_{\eta}(\phi) \frac{E_{b \eta}\left(T_{b}\right)}{E_{b}\left(T_{b}\right)} d \eta=\sum_{j=1}^{n} a_{j} C_{j}$

$1^{\text {st }}$ inverse moment ("Rosseland mean" of the absorption cross-section):

$$
\frac{1}{C_{R}\left(\phi, T_{b}\right)}=\mu_{-1}\left(\phi, T_{b}\right)=\int_{0}^{\infty} \frac{1}{C_{\eta}(\phi)} \frac{E_{b \eta}\left(T_{b}\right)}{E_{b}\left(T_{b}\right)} d \eta=\sum_{j=1}^{n} \frac{a_{j}}{C_{j}}
$$

Graphical representation of the SLW modelling of the first and first inverse moments by the histogram reordered absorption cross-section is shown in Fig. 2. The gray gas weights are calculated as $a_{j}\left(\phi, T_{b}\right)=F\left(\tilde{C}_{j}, \phi, T_{b}\right)-F\left(\tilde{C}_{j-1}, \phi, T_{b}\right)$. The terms "Planck mean" and "Rosseland 
mean" are used here conditionally, because they resemble the traditional Planck and Rosseland mean values of the absorption coefficient.

The ALBDF used in the SLW model is calculated using the high-resolution HITEMP 2010 spectroscopic database [13]. The ALBDF has been traditionally represented through one of two methods [21]: i) mathematical correlations, or $i$ ) stored tabulated form in a look-up table. It has been shown that the best accuracy can be achieved if the analytical expressions for the SLW models are evaluated with the full data of the look-up table which has nearly LBL accuracy. The look-up table presents the values of the ALBDF $F_{i}$ for discrete values of absorption crosssection $C_{i}, i=1,2, \ldots, N$, where $N=71$ for the look-up table published in [21]. The discrete look-up table data for the ALBDF for continuous SLW models [2] can be integrated analytically if a linear dependence of the ALBDF is assumed between finely-resolved tabulated points:

$$
\begin{aligned}
& C_{P}\left(\phi, T_{b}\right)=\mu_{1}\left(\phi, T_{b}\right)=\int_{0}^{\infty} C \frac{\partial F\left(C, \phi, T_{b}\right)}{\partial C} d C=\sum_{j=1}^{N}\left(F_{j}-F_{j-1}\right)\left(C_{j}+C_{j-1}\right) / 2 \\
& \frac{1}{C_{R}\left(\phi, T_{b}\right)}=\mu_{-1}\left(\phi, T_{b}\right)=\int_{C_{\min }}^{C_{\max }} \frac{1}{C} \frac{\partial F\left(C, \phi, T_{b}\right)}{\partial C} d C=\sum_{j=1}^{N} \frac{\left(F_{j}-F_{j-1}\right)}{\left(C_{j}+C_{j-1}\right)} \ln \left(\frac{C_{j}}{C_{j=1}}\right)
\end{aligned}
$$
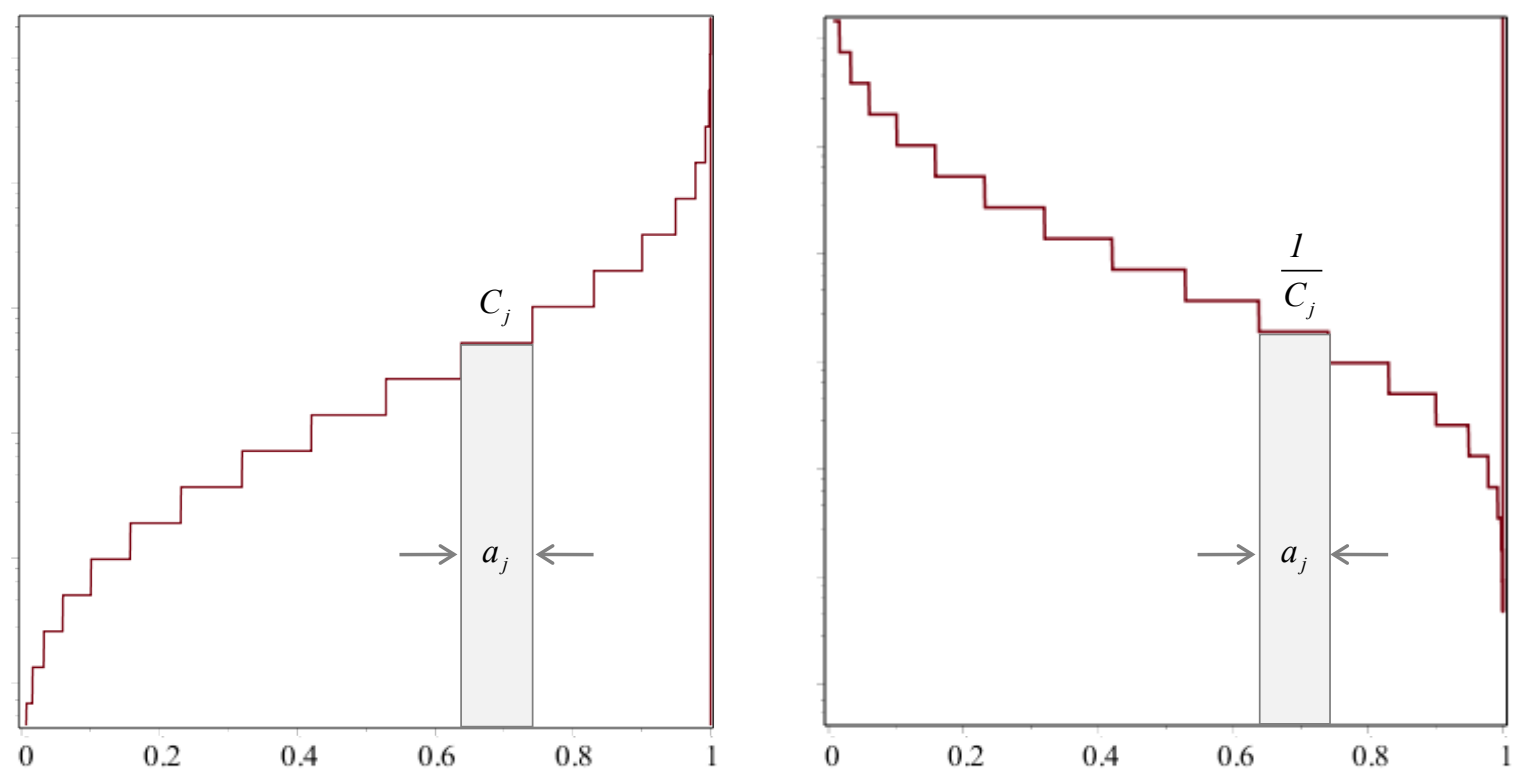

Figure 2. SLW modelling of the Planck mean and Rosseland mean absorption cross-sections. The area under the stepwise graph is numerically equal to $C_{P}$ and $1 / C_{R}$, respectively. 
These equations provide the best accuracy provided by the look-up table. It should be noted that the Rosseland mean of the gas absorption cross-section is not a characterization of the gas absorption spectrum in general, but rather of the SLW model histogram gray gas spectrum only excluding the clear gas (transparent spectral windows) [2].

\section{SLW modeling of the scaling coefficient}

Application of the scaled SLW models given by Eqs. (11) - (14) to Eq. (9) yields the following expressions used for the explicit calculation of the local scaling coefficients

$$
\begin{aligned}
& u_{P}^{l o c}=\frac{C_{P}\left(\phi_{l o c}, T_{l o c}\right)}{C_{P}\left(\phi_{r e f}, T_{l o c}\right)} \\
& u_{R}^{l o c}=\frac{C_{R}\left(\phi_{l o c}, T_{l o c}\right)}{C_{R}\left(\phi_{r e f}, T_{l o c}\right)}
\end{aligned}
$$

The sense of this scaling expression is that if the absorption spectra at different thermodynamic states are scaled, then they are scaled in the same manner as their Planck or Rosseland means.

From Eqs. (5) and (15), the similarity relationship for any local thermodynamic state follows:

$$
\frac{C_{\eta}\left(\phi_{l o c}\right)}{C_{P}\left(\phi_{l o c}, T_{l o c}\right)}=\frac{C_{\eta}\left(\phi_{r e f}\right)}{C_{P}\left(\phi_{r e f}, T_{l o c}\right)}
$$

Therefore, the "normalized" spectrum can be defined as

$$
\bar{C}_{\eta}=\frac{C_{\eta}\left(\phi_{r e f}\right)}{C_{P}\left(\phi_{r e f}, T_{l o c}\right)}
$$

which is invariant for any gas local thermodynamic state. Then the spectrum at any local state can be found as

$$
C_{\eta}\left(\phi_{l o c}\right)=C_{P}\left(\phi_{l o c}, T_{l o c}\right) \bar{C}_{\eta}
$$

It should be noted that the Scaled SLW model presented can also be developed in terms of the gas absorption coefficient $\kappa_{\eta}(\phi)=N(\phi) Y C_{\eta}(\phi)$, where $N$ is the gas molar density, rather than the gas absorption cross-section $C_{\eta}(\phi)$. The scaled spectrum assumption in this case is written as

$$
\kappa_{\eta}\left(\phi_{l o c}\right)=u_{l o c} \kappa_{\eta}\left(\phi_{r e f}\right)
$$

and the $s^{\text {th }}$ moment of gas absorption coefficient is defined as 


$$
\mu_{s}\left(\phi, T_{b}\right)=\int_{0}^{\infty}\left[\kappa_{\eta}(\phi)\right]^{s} \frac{E_{b \eta}\left(T_{b}\right)}{E_{b}\left(T_{b}\right)} d \eta
$$

The scaling coefficients following this development can then be defined as

$$
\begin{aligned}
& u_{P}=\frac{\kappa_{P}\left(\phi_{l o c}, T_{b}\right)}{\kappa_{p}\left(\phi_{r e f}, T_{b}\right)} \\
& u_{R}=\frac{\kappa_{R}\left(\phi_{l o c}, T_{b}\right)}{\kappa_{R}\left(\phi_{r e f}, T_{b}\right)}
\end{aligned}
$$

where $\kappa_{P}\left(\phi, T_{b}\right)$ and $\kappa_{R}\left(\phi, T_{b}\right)$ are the SLW modified Planck mean and Rosseland mean absorption coefficients, respectively.

It should be noted that formulation of the Scaled SLW model in terms of absorption coefficient is similar but not equivalent to the scaled SLW model in terms of gas absorption cross-section. The scaled SLW model in terms of absorption coefficient was not investigated in this paper.

\section{The Scaled SLW model}

The scaling assumption outlined previously yields a scaled histogram absorption cross-section which, for any spectral location, is defined by $C_{j}^{l o c}=u^{l o c} C_{j}^{r e f}$ from the arbitrarily chosen subdivision into reference supplemental absorption cross-sections $C_{j}^{\text {ref }}$.

The wavenumber gray gas intervals defined by the reference supplemental cross-sections and absorption cross-section at the reference state for the scaled absorption cross-section defined by Eq. (5) remain the same at all thermodynamic states, as described by Eq. (24) and shown in Fig. 3:

$$
\Delta_{j}=\left\{\eta: \tilde{C}_{j-1}^{r e f}<C_{\eta}\left(\phi_{r e f}\right)<\tilde{C}_{j}^{r e f}\right\}=\left\{\eta: u^{l o c} \tilde{C}_{j-1}^{r e f}<C_{\eta}\left(\phi_{l o c}\right)<u^{l o c} \tilde{C}_{j}^{r e f}\right\}
$$

Therefore, integration of the spectral RTE over wavenumber intervals $\Delta_{j}$ yields the gray gas RTE without additional Leibnitz terms of integration:

$\frac{\partial I_{j}(s)}{\partial s}=-\kappa_{j}(s) I_{j}(s)+a_{j}(s) \kappa_{j}(s) I_{b}(T(s))$

Here, the local gray gas absorption coefficients at location $s$ are defined as

$$
\kappa_{j}(s)=N(s) Y(s) u(s) C_{j}^{r e f}
$$

The local gray gas weights can be calculated using two different approaches. In what is termed here Method 1, the weights are determined with the reference supplemental cross-sections and the reference thermodynamic state: 
$a_{j}(s)=F\left(\tilde{C}_{j}^{r e f}, \phi_{r e f} T_{l o c}\right)-F\left(\tilde{C}_{j-1}^{r e f}, \phi_{r e f} T_{l o c}\right)$

Alternatively, the weights can be calculated with the scaled supplemental cross-sections and the local thermodynamic state, denoted Method 2:

$a_{j}(s)=F\left(u^{l o c} \tilde{C}_{j}^{r e f}, \phi_{l o c}, T_{l o c}\right)-F\left(u^{l o c} \tilde{C}_{j-1}^{r e f}, \phi_{l o c}, T_{l o c}\right)$

The difference between calculation using the reference or local supplemental cross-sections (Methods 1 and 2, respectively) is visualized in Fig. 3. Theoretically, for truly scaled spectra, these two methods for calculating the gray gas weights should produce equivalent results since they represent the same fraction of the Planck spectral emissive power at the same blackbody temperature, $T_{b}=T_{l o c}$. However, because real spectra are not perfectly scaled Eqs. (27) and (28) yield, in general, different results. This will be demonstrated in a section to follow.

\section{Continuous limit of the Scaled SLW model}

It is useful at this point to confirm that the Scaled SLW model approaches the continuous limit with an increase in number of gray gases. This is done by first defining the intensity at the supplemental absorption cross-section $\tilde{C}_{j}^{\text {ref }}$ using the equation:

$I_{j}=I\left(\tilde{C}_{j}^{r e f}\right) \Delta C_{j}^{r e f}, \Delta C_{j}^{r e f}=\tilde{C}_{j}^{r e f}-\tilde{C}_{j-1}^{r e f}$

Substitution of the gray gas absorption coefficients Eq. (26) and the gray gas weights Eq. (27), the gray gas RTE Eq. (25) can then be written as

$$
\frac{\partial I\left(\tilde{C}_{j}^{r e f}\right)}{\partial s}=-N Y u C_{j}^{r e f} I\left(\tilde{C}_{j}^{r e f}\right)+\frac{F\left(\tilde{C}_{j}^{r e f}, \phi_{r e f}, T\right)-F\left(\tilde{C}_{j-1}^{r e f}, \phi_{r e f}, T\right)}{\Delta C_{j}^{r e f}} N Y u C_{j}^{r e f} I_{b}(T)
$$

Here, $T=T(s)$ is the local gas temperature and $N=N(s), Y=Y(s)$, and $u=u(s)$ are the corresponding local values of gas molar density, mole fraction, and scaling coefficient, respectively. With an increase in the number of gray gases in the model, the gray gas supplemental cross-sections approach the continuous variable $\tilde{C}_{j}^{r e f} \rightarrow \xi$, and the finite increment in Eq. (30) becomes the continuous limit of the Scaled SLW model:

$$
\frac{\partial I(\xi)}{\partial s}=-N Y u \xi I(\xi)+\frac{\partial F\left(\xi, \phi_{r e f}, T\right)}{\partial \xi} N Y u \xi I_{b}(T)
$$

The total radiation intensity is found by

$I(s)=\int_{0}^{\infty} I_{\eta}(s) d \eta=\sum_{j=0}^{n} I_{j}=\sum_{j=0}^{n} I\left(\tilde{C}_{j}^{r e f}\right) \Delta C_{j}^{r e f}=\int_{0}^{1} I(\xi) d \xi$

The geometric meaning of the derivative term $\partial F\left(\xi, \phi_{r e f}, T\right) / \partial \xi$ in Eq. (31) is illustrated in Fig. 3 , where the derivative is seen as the slope of the tangent line to the ALBDF curve. 


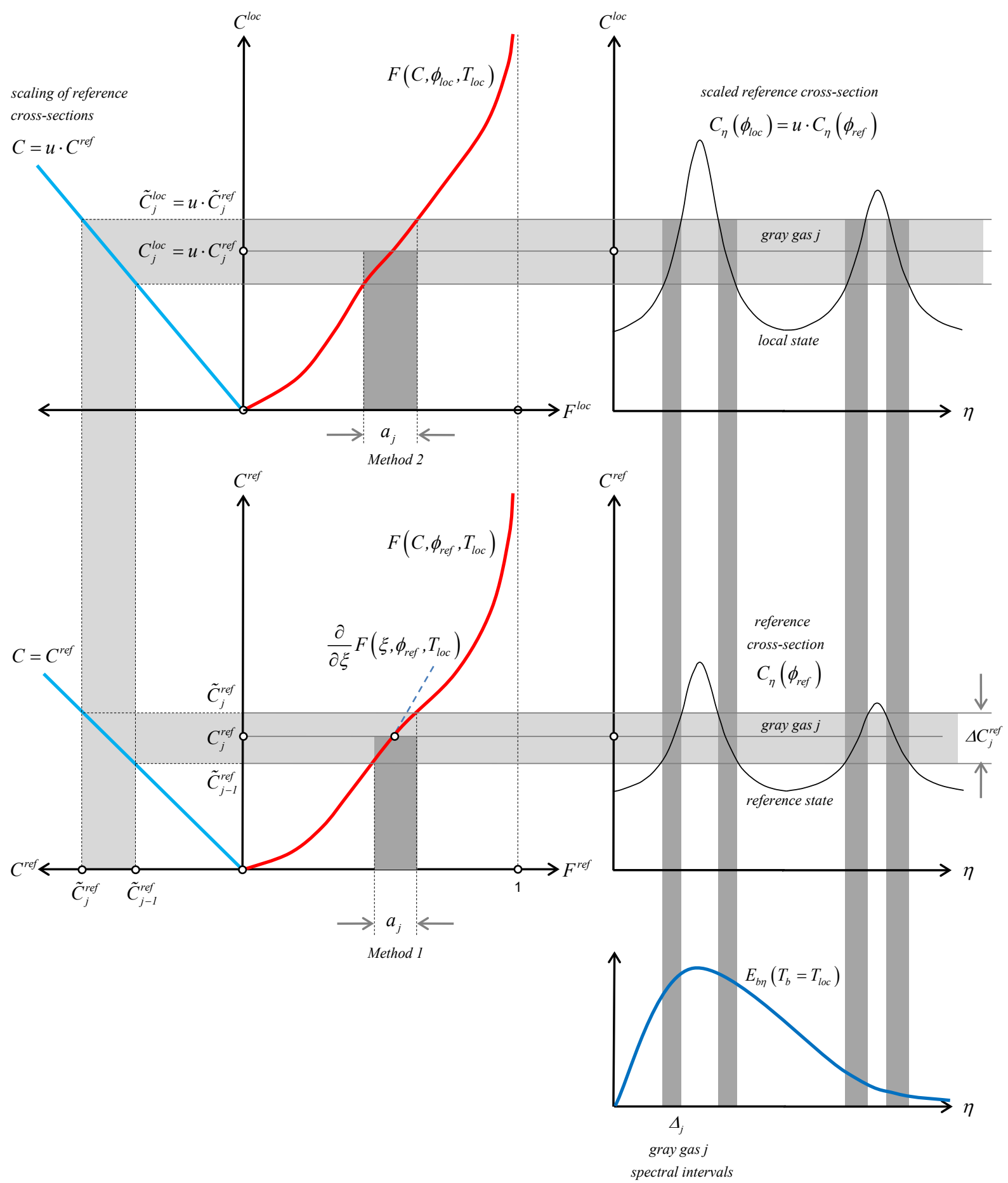

Figure 3. Graphical representation of spectral scaling in the Scaled SLW spectral model.

\section{Theoretical justification of the Scaled SLW model by the $\ell$-distribution method}

The choice of the gas reference thermodynamic state and the order of the moments of the absorption cross-section for construction of the proposed scaled spectral model are arbitrary, 
which can be used to advantage for improvement of the model's accuracy. To make a better selection (preference) between the Planck and Rosseland mean approaches, one may apply recent developments in the modelling of radiation transfer in non-uniform media with the help of a new approach termed the $\ell$-distribution method [22, 23], which is based on application of statistical theory to narrow band modelling of radiative transfer.

The $\ell$-distribution method is developed as a narrow band model for prediction of radiative transfer along uniform and non-uniform gaseous paths [22]. However, in general, the method is not restricted to narrow bands, and it can thus be extended to the full spectrum. Therefore, one may modify here the main concepts of the $\ell$-distribution method needed for this application directly to the full spectrum version. The $\ell$-distribution theory summarized here will provide further theoretical justification for the proposed scaling relations of Eqs. (15) and (16).

The total transmissivity of a uniform path $L$ is obtained by

$$
\tau(L)=\int_{0}^{\infty} e^{-\kappa_{\eta}(\eta, \phi) L} \frac{E_{b \eta}\left(T_{b}\right)}{E_{b}\left(T_{b}\right)} d \eta
$$

where the integral extends over all wavenumbers for which the absorption coefficient is strictly positive, e.g., using the same intervals as for $\kappa_{R}$. The total transmissivity $\tau(L)$ is as a strictly decreasing function of pathlength $L$, and is thus invertible. Its inverse $\ell$ can be defined as solution of the relation:

$$
\ell[\tau(L)]=L
$$

The assumption of the path-dependent scaling approach is formulated in the following way: For any length $L_{1}$ inside the gas at thermodynamic state $\phi_{1}$ the total transmissivity is preserved by the scaling coefficient

$$
\int_{0}^{\infty} E_{b \eta}\left(T_{b}\right) e^{-\kappa_{\eta}\left(\phi_{1}\right) L_{1}} d \eta=\int_{0}^{\infty} E_{b \eta}\left(T_{b}\right) e^{-\kappa_{\eta}\left(\phi_{2}\right) u\left(\phi_{1}, \phi_{2}, L_{1}\right) L_{1}} d \eta
$$

which establishes the scaling relationship between the absorption spectra at states $\phi_{1}$ and $\phi_{2}$

$$
\kappa_{\eta}\left(\phi_{1}\right)=u\left(\phi_{1}, \phi_{2}, L_{1}\right) \kappa_{\eta}\left(\phi_{2}\right)
$$

Here, the scaling coefficient $u\left(\phi_{1}, \phi_{2}, L_{1}\right)$ is path-dependent, in contrast to the scaling coefficient in Eq. (3). However, it is independent of wavenumber $\eta$, and $\kappa_{\eta}\left(\phi_{2}\right)$ is the absorption coefficient at any arbitrary state $\phi_{2}$. (In some sense it can play a role of the reference state.) If the non-uniform path $L$ consists of two uniform layers of length $L_{1}$ and $L_{2}$, then the scaling coefficient in $[22,23]$ is defined as

$$
u\left(\phi_{1}, \phi_{2}, L_{1}\right)=\ell_{2}\left(\tau_{1}\left(L_{1}\right)\right) / L_{1}
$$


It was shown in ref. $[22,23]$ that a rough though relevant approximation of the numerator in the right-hand side of Eq. (37) is

$$
\ell_{2}\left(\tau_{1}\left(L_{1}\right)\right)=\frac{\kappa_{P, 1}}{\kappa_{P, 2}} \frac{\beta_{1}}{\beta_{2}} L_{1}-\frac{\kappa_{P, 1}}{\kappa_{P, 2}}\left(1-\frac{\beta_{1}}{\beta_{2}}\right) \frac{\beta_{1}}{\pi \kappa_{P, 1}}\left(1-\sqrt{1+\frac{2 \pi \kappa_{P, 1} L_{1}}{\beta_{1}}}\right)
$$

where the Planck and Rosseland mean absorption coefficient (first and first inverse moments) are calculated by including the weight function for spectral integration $(m=1,2)$ :

$$
\begin{gathered}
\kappa_{P, m}=\int_{0}^{\infty} \kappa_{\eta}\left(\phi_{m}\right) \frac{E_{b \eta}\left(T_{b}\right)}{E_{b}\left(T_{b}\right)} d \eta \\
\kappa_{R, m}=\int_{0}^{\infty} \frac{1}{\kappa_{\eta}\left(\phi_{m}\right)} \frac{E_{b \eta}\left(T_{b}\right)}{E_{b}\left(T_{b}\right)} d \eta
\end{gathered}
$$

and the parameter $\beta$ is defined as

$$
\beta_{m}=\pi\left(\frac{\kappa_{P, m}}{\kappa_{R, m}}-1\right)^{-1}
$$

It should be noted that the Rosseland mean (first inverse moment) is not usually defined for gases in the full spectrum because of spectral windows of the gas absorption coefficient which prohibit division by $\kappa_{\eta}=0$ in Eq. (40). Therefore, the Rosseland mean is treated here exclusively in the context of the Generalized SLW model which excludes transparent windows from its definition [2].

If $2 \pi \kappa_{P, 1} L_{1} / \beta_{1} \ll 1$ (as can be the case of an optically thin layer) in Eq. (38), then a linear approximation of the square root can be taken, $\sqrt{1+x} \approx 1+x / 2$, and the equation reduces to

$$
\ell_{2}\left(\tau_{1}\left(L_{1}\right)\right) \approx \frac{\kappa_{P, 1}}{\kappa_{P, 2}} L_{1}
$$

The scaling coefficient for the optically thin case thus becomes

$$
u\left(\phi_{1}, \phi_{2}, L_{1}\right)=\frac{\ell_{2}\left(\tau_{1}\left(L_{1}\right)\right)}{L_{1}} \approx \frac{\kappa_{P, 1}\left(T_{b}\right)}{\kappa_{P, 2}\left(T_{b}\right)}
$$

which corresponds to the scaling coefficient $u_{P}$ defined by Eq. (15).

If $\kappa_{P} \ll \kappa_{R}$ (which is observed for gases [2]) then $\beta_{m} \approx \pi \kappa_{R, m} / \kappa_{P, m}$ and the scaling coefficient can be approximated as the ratio of Rosseland means: 
$u\left(\phi_{1}, \phi_{2}, L_{1}\right)=\frac{\ell_{2}\left(\tau_{1}\left(L_{1}\right)\right)}{L_{1}} \approx \frac{\kappa_{R, 1}\left(T_{b}\right)}{\kappa_{R, 2}\left(T_{b}\right)}$

which corresponds to the scaling coefficient $u_{R}$ defined by Eq. (16). Thus, the application of previously published $\ell$-distribution theory confirms and supports the theoretical basis of Eqs. (15) and (16) for calculation of the scaling coefficients in the Scaled SLW model. Further, this development underlines the significant advantage of the scaling coefficient being determined explicitly, which is an important feature of the current model's theoretical development.

\section{Relationship to the scaled FSK method}

The current theoretical construction of the scaling coefficient can be related to prior work using the scaled spectrum assumption in the context of the FSK method. The exponential terms in Eq. (1) for the scaling coefficient of the FSK method may be replaced by their linear approximation of exponential functions:

$$
\begin{aligned}
& e^{-\kappa_{\eta}(\eta, \phi) L_{m}} \approx 1-\kappa_{\eta}(\eta, \phi) L_{m} \\
& e^{-\kappa_{\eta}\left(\eta, \phi_{0}\right) u\left(\phi, \phi_{0}\right) L_{m}} \approx 1-\kappa_{\eta}\left(\eta, \phi_{0}\right) u\left(\phi, \phi_{0}\right) L_{m}
\end{aligned}
$$

These approximations are then substituted in Eq. (1) and simplified

$$
\int_{0}^{\infty} \kappa_{\eta}(\eta, \phi) I_{b \eta}\left(T_{0}\right) d \eta=\int_{0}^{\infty} \kappa_{\eta}\left(\eta, \phi_{0}\right) u\left(\phi, \phi_{0}\right) I_{b \eta}\left(T_{0}\right) d \eta
$$

The scaling coefficient can then be factored

$$
\int_{0}^{\infty} \kappa_{\eta}(\eta, \phi) I_{b \eta}\left(T_{0}\right) d \eta=u\left(\phi, \phi_{0}\right) \int_{0}^{\infty} \kappa_{\eta}\left(\eta, \phi_{0}\right) I_{b \eta}\left(T_{0}\right) d \eta
$$

If both sides of the relation above are divided by the total Planck emissive power at the reference temperature

$$
\frac{1}{I_{b}\left(T_{0}\right)} \int_{0}^{\infty} \kappa_{\eta}(\eta, \phi) I_{b \eta}\left(T_{0}\right) d \eta=u\left(\phi, \phi_{0}\right) \frac{1}{I_{b}\left(T_{0}\right)} \int_{0}^{\infty} \kappa_{\eta}\left(\eta, \phi_{0}\right) I_{b \eta}\left(T_{0}\right) d \eta
$$

and the definition of the modified Planck mean absorption coefficient is applied

$$
\kappa_{P}\left(\phi, T_{0}\right)=u\left(\phi, \phi_{0}\right) \kappa_{P}\left(\phi_{0}, T_{0}\right)
$$

one may solve for the scaling coefficient

$$
u\left(\phi, \phi_{0}\right)=\frac{\kappa_{P}\left(\phi, T_{0}\right)}{\kappa_{P}\left(\phi_{0}, T_{0}\right)}
$$


This expression reveals that the scaled Planck SLW model in terms of absorption coefficient is equivalent to the linear approximation of the scaled FSK method. This result is also equivalent to Eq. (43), which corresponds to the optically thin approximation in the $\ell$-distribution method. Invoking the linear approximation yields the advantage of an explicit calculation of the scaling coefficient, as mentioned previously. The accuracy of the assumption under this development will now be explored.

\section{MODEL PREDICTIONS}

The Scaled SLW model as developed in the foregoing sections has been exercised through prediction of radiative transfer in example problems designed to test the scaled spectrum model assumption. Predictions are explored using both the Planck mean- and Rosseland mean-based scaling coefficient. These predictions are compared to line-by-line (LBL) benchmark predictions, and to predictions made with the SLW Reference Approach and the recently published Rank Correlated SLW model. This allows the comparison of predictions using both correlated and scaled spectrum assumptions. In all cases the same spectral database has been used for the line-by-line calculations and the generation of the ALBDF used in the Scaled SLW, Rank Correlated SLW, and SLW Reference Approach model predictions. The tabulated form of the ALBDF has been used for the Scaled SLW model, Rank Correlated model, and SLW Reference Approach predictions.

\section{Example 1}

Consider the prediction of the divergence of total net radiative flux in a plane-parallel layer of thickness $L$ bounded by black walls filled with a mixture of water vapor and carbon dioxide. The gas temperature and species mole fractions have a parabolic profile defined by

$$
\begin{aligned}
& T(x)=4000 x(L-x) / L^{2}+800, \mathrm{~K} \\
& Y_{\mathrm{H}_{2} \mathrm{O}}(x)=0.8 x(L-x) / L^{2}+0.12 \\
& Y_{\mathrm{CO}_{2}}(x)=0.4 x(L-x) / L^{2}+0.06
\end{aligned}
$$

and are illustrated graphically in Fig. 4. Four different cases for the gas layer thickness are considered in this example problem, $L=0.01,0.1,1.0$, and $5.0 \mathrm{~m}$. This permits the identification of any dependence of the models' accuracy on optical depth. In all cases, the maximum temperature in the gas layer $T_{\max }=1800 \mathrm{~K}$ occurs at the middle point of the layer and the minimum temperature $T_{\min }=T_{0}=T_{L}=800 \mathrm{~K}$ is at the walls. The difference between maximum and minimum temperature in the layer is $\Delta T=1000 \mathrm{~K}$, which, for the different layer thicknesses explored, results in substantially different spatial temperature gradients. The average gas temperature of the layer for all cases is $T_{\text {ave }}=1467 \mathrm{~K}$. The ratio of gas mole fractions, $\mathrm{H}_{2} \mathrm{O}$ and $\mathrm{CO}_{2}$, is spatially constant for this example at $Y_{\mathrm{H}_{2} \mathrm{O}} / Y_{\mathrm{CO}_{2}}=2$. 


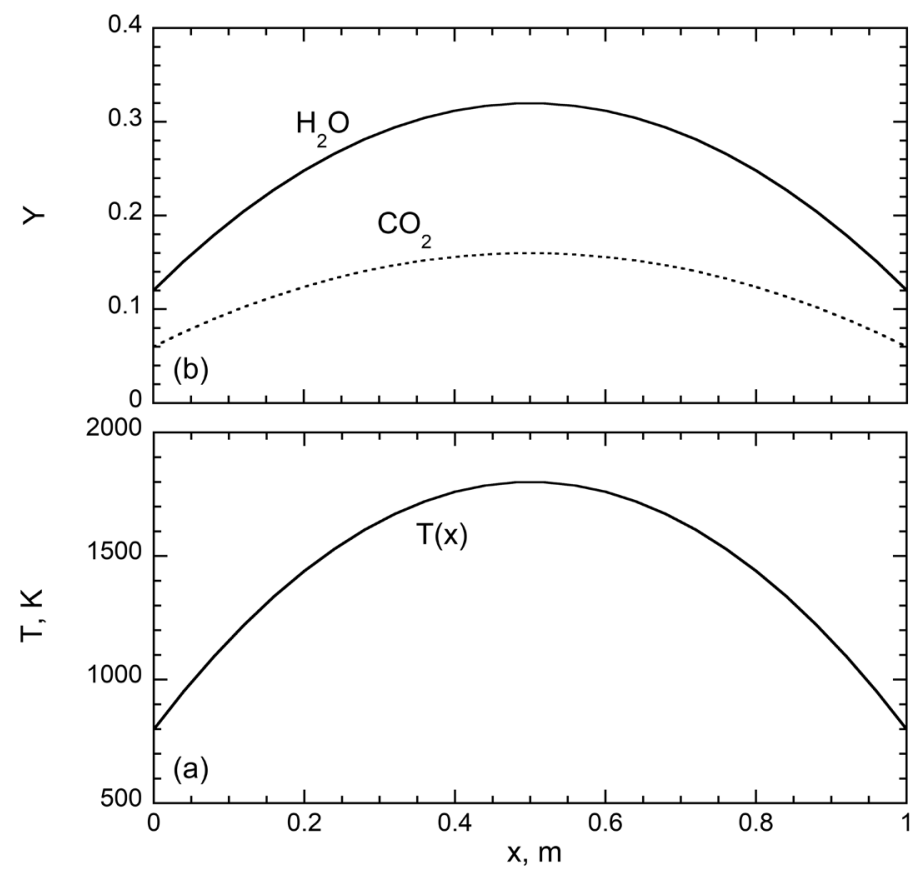

Figure 4. Profiles of a) temperature, and b) gas mole fraction for Example 1.

Prediction of the total divergence of the net radiative flux is performed using the analytical solution for the multilayer SLW solution method [24], with the Scaled SLW Planck mean (SPSLW) and Scaled SLW Rosseland mean (SR-SLW) models developed here, the Rank Correlated SLW model (RC-SLW) [3], and the SLW Reference Approach (SLW-RA) [6]. The mixture of $\mathrm{H}_{2} \mathrm{O}$ and $\mathrm{CO}_{2}$ in the SLW methods is treated as a single gas using the SLW multiplication approach described in [25]. The values of the minimum and maximum absorption cross-section, $C_{\min }=3 \times 10^{-4} \mathrm{~m}^{2} / \mathrm{mol}$ and $C_{\max }=60 \mathrm{~m}^{2} / \mathrm{mol}$, have been chosen to effectively cover the entire gas mixture absorption cross-section and to maintain the scaled absorption cross-sections in the range of the cross-sections in the ALBDF look-up table [21]. 25 gray gases have been used in all SLW model predictions to ensure that prediction error is not caused by discretization into gray gases. The line-by-line (LBL) benchmark predictions are performed with the same multilayer exact solution as that employed in the SLW method solutions, with spectral integrations performed using a summation over spectral intervals. The high resolution spectra based on the HITEMP2010 [13] spectral database for $\mathrm{H}_{2} \mathrm{O}$, and the CDSD-4000 [26] database for $\mathrm{CO}_{2}$ were used with spectral discretization $\Delta \eta=0.01 \mathrm{~cm}^{-1}$. More details about these spectral databases can be found in [27]. Detailed comparisons, not reported here, have shown that the use of the CDSD-4000 database instead of HITEMP2010 for $\mathrm{CO}_{2}$ in the construction of the SLW model database does not provide significant differences in full spectrum uniform scenarios. 
Calculations are first made in this example problem for the case of layer thickness $L=1.0 \mathrm{~m}$ to compare the methods for determining the local gray gas weights according to Eq. (27) using Method 1, and according to Eq. (28) using Method 2. This case is also used to consider in a preliminary way the influence of the choice of the gas reference temperature $T_{\text {ref. }}$. Two the most widely used choices for gas reference temperature are compared, namely 1) the spatially averaged temperature, calculated as

$$
T_{\text {ref }}=T_{\text {ave }}=\frac{1}{L} \int_{0}^{L} T(x) d x,
$$

and 2) the maximum temperature in the medium $T_{r e f}=T_{\max }$, which, for this example is $T_{\max }=$ 1800K. Comparison is made in Fig. 5a of the total divergence of the local net radiative flux, $Q(x)$, for the scaled model based on Planck mean (SP-SLW), and in Fig. $5 \mathrm{~b}$ for the scaled model based on Rosseland mean (SR-SLW). Although this is only one example problem, the predictions suggest that the combination of Method 1 used for calculation of the gray gas weights and the use of the maximum temperature as reference temperature yield the most accurate results. On this basis Method 1 will be used in all predictions hereafter. The optimal reference temperature will be explored at length in sections to follow.
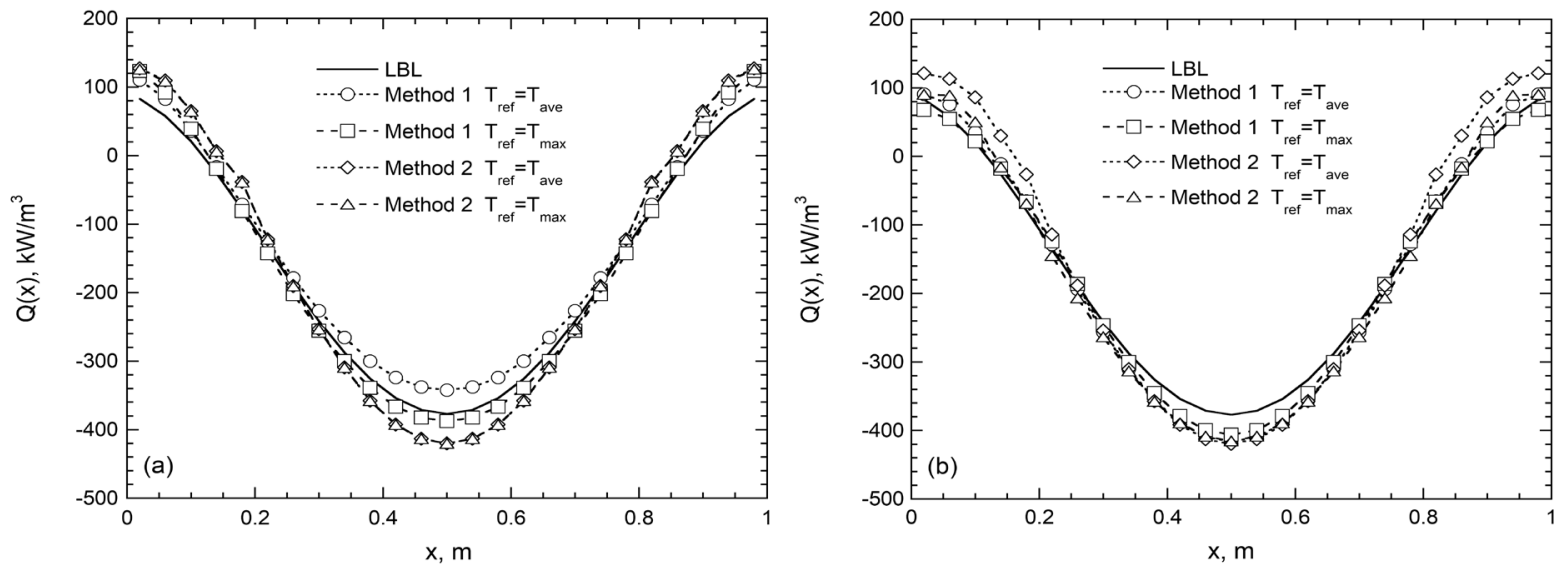

Figure 5. Predicted total divergence of the local net radiative flux for a layer thickness $L=1.0 \mathrm{~m}$ using a) the SP-SLW model, and b) the SR-SLW model.

For better evaluation and quantitative comparison of the performance of different methods, the local and total absolute relative error of prediction of the total divergence of the net radiative flux are defined, respectively, as

$$
L E(x)=\left|Q_{S L W}(x)-Q_{L B L}(x)\right| / \max \left|Q_{L B L}(x)\right|
$$


and

$$
T E=\int_{0}^{L}\left|Q_{S L W}(x)-Q_{L B L}(x)\right| d x / \int_{0}^{L}\left|Q_{L B L}(x)\right| d x
$$

These quantities are calculated and presented to provide comparison of the prediction by different methods.

The results for prediction of the total divergence of net radiative flux are shown in Fig. 6a - 6d for the four layer thicknesses studied, $L=0.01 \mathrm{~m}, 0.1 \mathrm{~m}, 1.0 \mathrm{~m}$, and $5.0 \mathrm{~m}$. The figure also illustrates the local absolute relative error $L E(x)$ as defined in Eq. (53). The maximum temperature in the domain was used as reference temperature $\left(T_{r e f}=T_{\max }\right)$ for the SP-SLW, SRSLW, and RA-SLW model predictions, and the average temperature was used for the blackbody source temperature $\left(T_{b}=T_{\text {ave }}\right)$ in the RC-SLW model predictions. The Rank Correlated SLW (RC-SLW) model is the most advanced correlated SLW model available [3]. Figure 6 shows that this model yields very accurate predictions. The accuracy for this model exhibits generally a modest increase in error as $L$ increases. The traditional SLW Reference Approach (RA-SLW) shows similar behavior. The Scaled Planck mean-based SLW model (SP-SLW) yields very good accuracy at low layer thicknesses, with the local error rising as $L$ increases. Finally, the Scaled Rosseland mean-based SLW model (SR-SLW) exhibits higher error at the extremes of low and high layer thickness, with minimum local error found for $L=1.0 \mathrm{~m}$. Interestingly, for the choice of reference temperature employed here $\left(T_{r e f}=T_{\max }\right)$ the Planck mean-based Scaled SLW model yields the best accuracy of the two Scaled SLW models.

Figure 7 illustrates the dependence of the total absolute relative error, $T E$, on reference temperature $T_{r e f}$ for the SP-SLW, SR-SLW, and RA-SLW models, and $T_{b}$ for the RC-SLW model for the conditions of Example 1. For this case the following observations may be made. The error in prediction for the traditional SLW Reference Approach (RA-SLW) decreased monotonically as $T_{\text {ref }}$ increases from the minimum to the maximum temperature in the domain. It appears that the $T_{r e f}=T_{\max }$ is the optimal choice of reference temperature for this model. This has been suggested previously [14, 28]. The Rank Correlated SLW (RC-SLW) model yields accurate predictions which are insensitive to the choice of source temperature, $T_{b}$. there appears to be slight increase in $T E$ with $T_{b}$ for the RC-SLW model, and the total error also appears to increase with increasing layer thickness.

Figure 7 reveals that the Planck mean-based Scaled SLW (SP-SLW) model exhibits a monotonically decreasing total error with increasing reference temperature at low layer thicknesses, with the best predictive accuracy found employing $T_{r e f}=T_{\max }$. However, for $L \geq$ $1.0 \mathrm{~m}$ there exists a local minimum in the total error's dependence on $T_{\text {ref. }}$. The local minimum error moves to lower reference temperature as the layer thickness increases; for $L=1.0 \mathrm{~m} T_{\text {ref }} \approx$ $1675 \mathrm{~K}$ yields the best solution, and for $L=5.0 \mathrm{~m} T_{r e f} \approx 1550 \mathrm{~K}$ is optimal. 

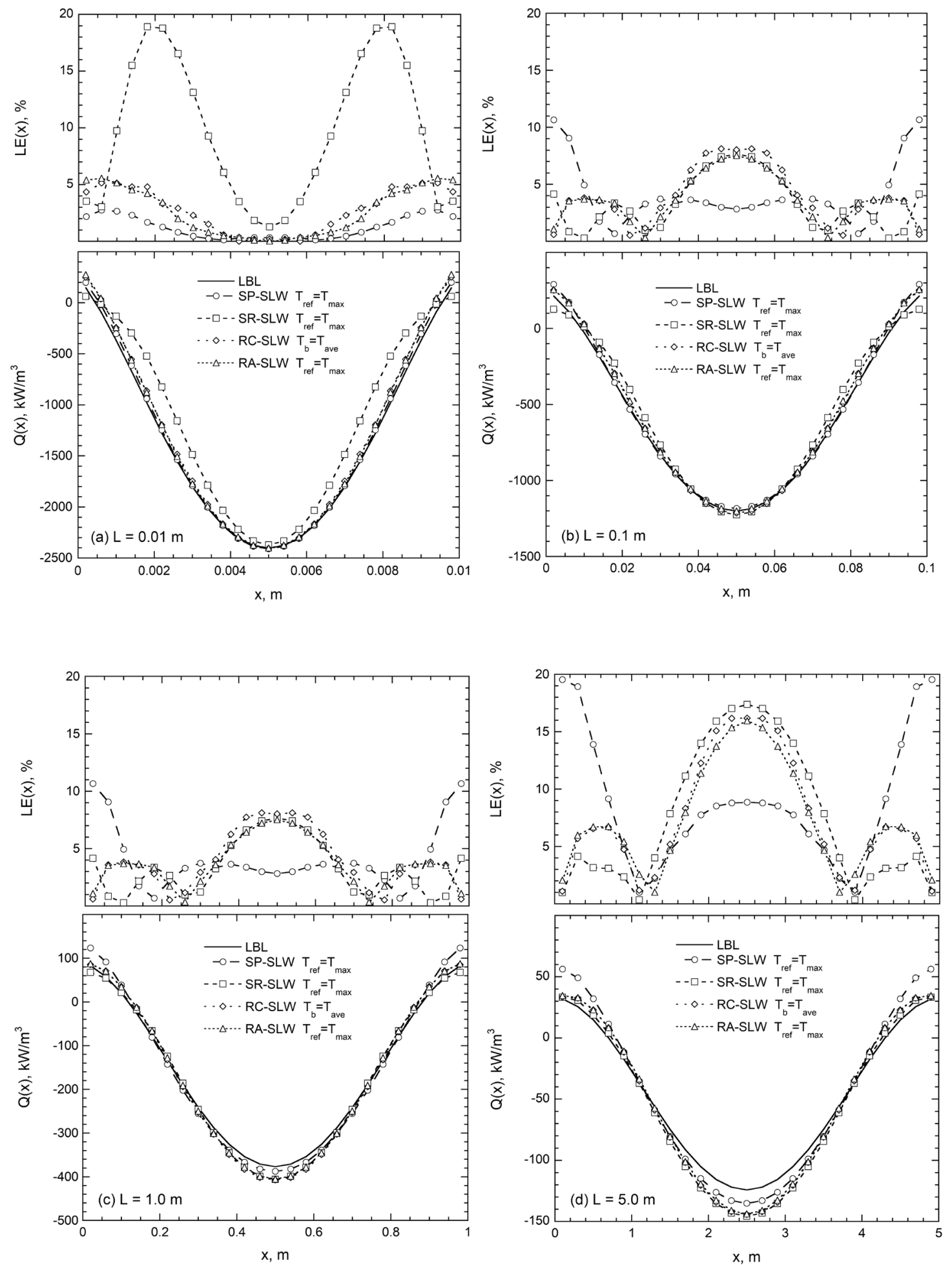

Figure 6. Predicted total divergence of the local net radiative flux $Q(x)$ and corresponding local relative error $L E(x)$ for the four layer thicknesses studied: a) $L=0.01 \mathrm{~m}$, b) $L=0.1 \mathrm{~m}$, c) $L=1.0$ $\mathrm{m}$, and d) $L=5.0 \mathrm{~m}$ 

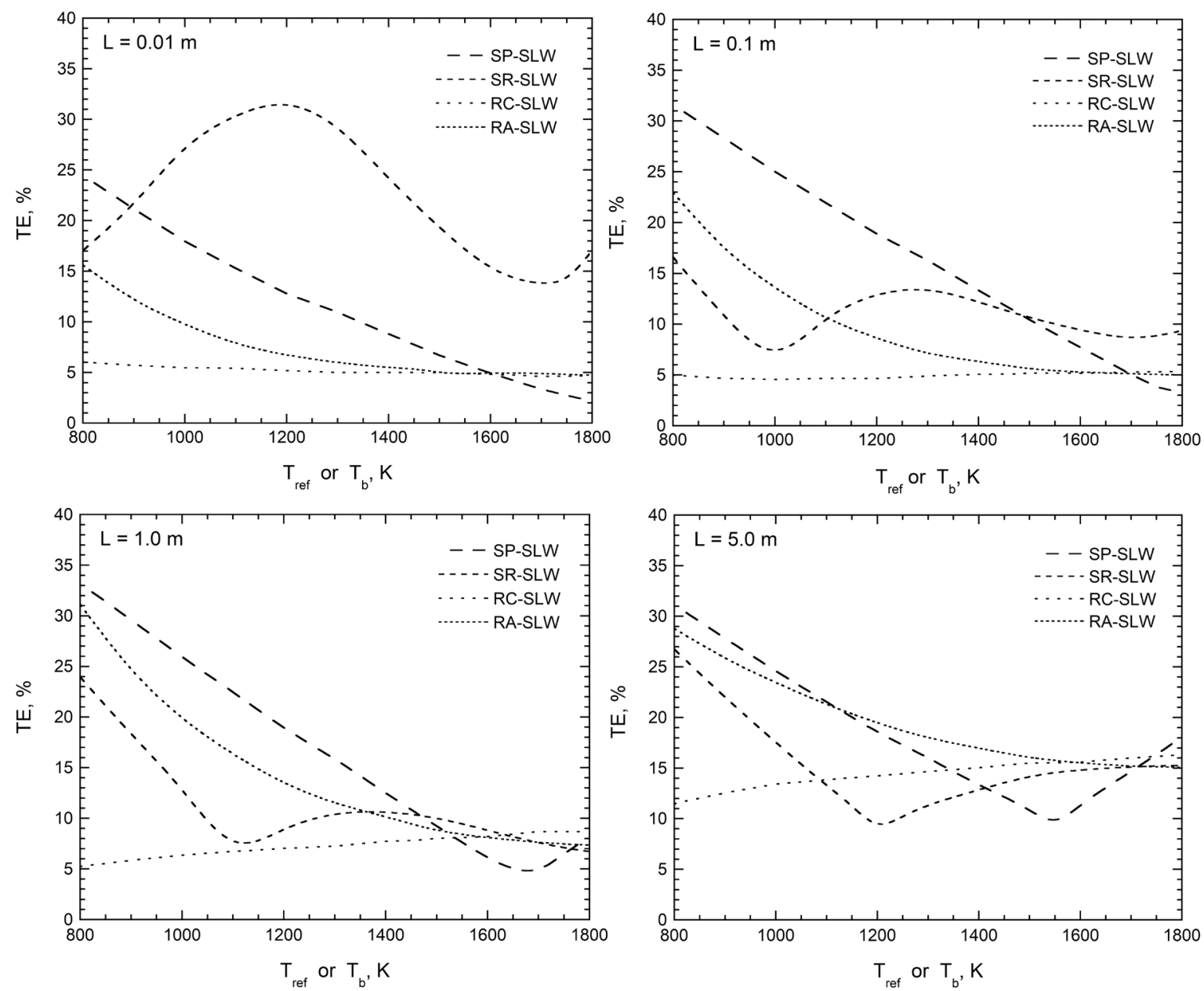

Figure 7. Predicted total relative absolute error as a function of reference temperature $T_{r e f}$ for the SP-SLW, SR-SLW, RA-SLW models; and $T_{b}$ for the RC-SLW model for the four layer thicknesses studied in Example 1.

The total relative error for the Rosseland mean-based Scaled SLW (SR-SLW) model predictions exhibits a considerably more complex dependence on reference temperature. For this Scaled SLW model the minimum total error for this example may be found somewhere between the minimum and maximum temperatures in the domain. Although the use of $T_{r e f}=T_{\max }$ for the SRSLW model will yield acceptable predictions, the complex dependence of $T E$ on $T_{\text {ref }}$ makes a more reasoned recommendation for the optimal reference temperature difficult to determine for this model.

It might also be mentioned that predictions have been made using $T_{r e f}$ (or $T_{b}$ for the RC-SLW model) outside the range of minimum and maximum temperature in the domain, which in some 
cases yield better accuracy than those utilizing $T_{\min } \leq T_{\text {ref }} \leq T_{\max }$. While this is an interesting finding, the theoretical basis for such a choice of reference temperature is unclear at this point, and deserves further investigation.

It is interesting to note that the more restrictive Scaled SLW models may yield predictions that are more accurate than the less restrictive Rank Correlated SLW model, provided an optimized reference temperature can be identified for use in the Scaled models. However, the Rank Correlated SLW model retains the significant advantage of requiring the specification of no reference state (temperature), and further, the RC-SLW model predictions are nearly insensitive to the source temperature, $T_{b}$.

There is theoretical justification for the use of $T_{r e f}=T_{\max }$ in the Scaled SLW model. The dependence of the mean absorption cross-sections on gas temperature $T$, which defines the gas thermodynamic state $\phi=\{T, Y, p\}$, is shown in Fig. 8. The gas absorption cross-section data of Fig. 8a reveals that $C_{P}\left(\phi, T_{b}\right)$ and $C_{R}\left(\phi, T_{b}\right)$ exhibit different dependence on gas temperature. As shown in Fig. 8b, this yields a strong variation in the ratio $C_{P}\left(\phi, T_{b}\right) / C_{R}\left(\phi, T_{b}\right)$ with gas temperature which reaches its minimum value at the highest temperature in the layer. The ratio $\kappa_{P} / \kappa_{R}$, which is proportional to $C_{P}\left(\phi, T_{b}\right) / C_{R}\left(\phi, T_{b}\right)$, appears in Eq. (41) defining the parameter $\beta$ of the $\ell$-distribution method [22,23]. According to the $\ell$-distribution method as applied to narrow bands, the value of gas temperature for which the parameter $\beta$ is maximum (i.e., the value of $\beta$ that corresponds to gas temperature for which the ratio $C_{P}\left(\phi, T_{b}\right) / C_{R}\left(\phi, T_{b}\right)$ is minimum) provides an optimum choice for the reference temperature. Theoretically, this fact has not yet been proven for global models. However, in the example considered here, it is consistent with narrow band observations; the ratio $C_{P}\left(\phi, T_{b}\right) / C_{R}\left(\phi, T_{b}\right)$ achieves its minimum at temperatures near $T=1800 \mathrm{~K}$, which is the maximum gas temperature in Example 1.
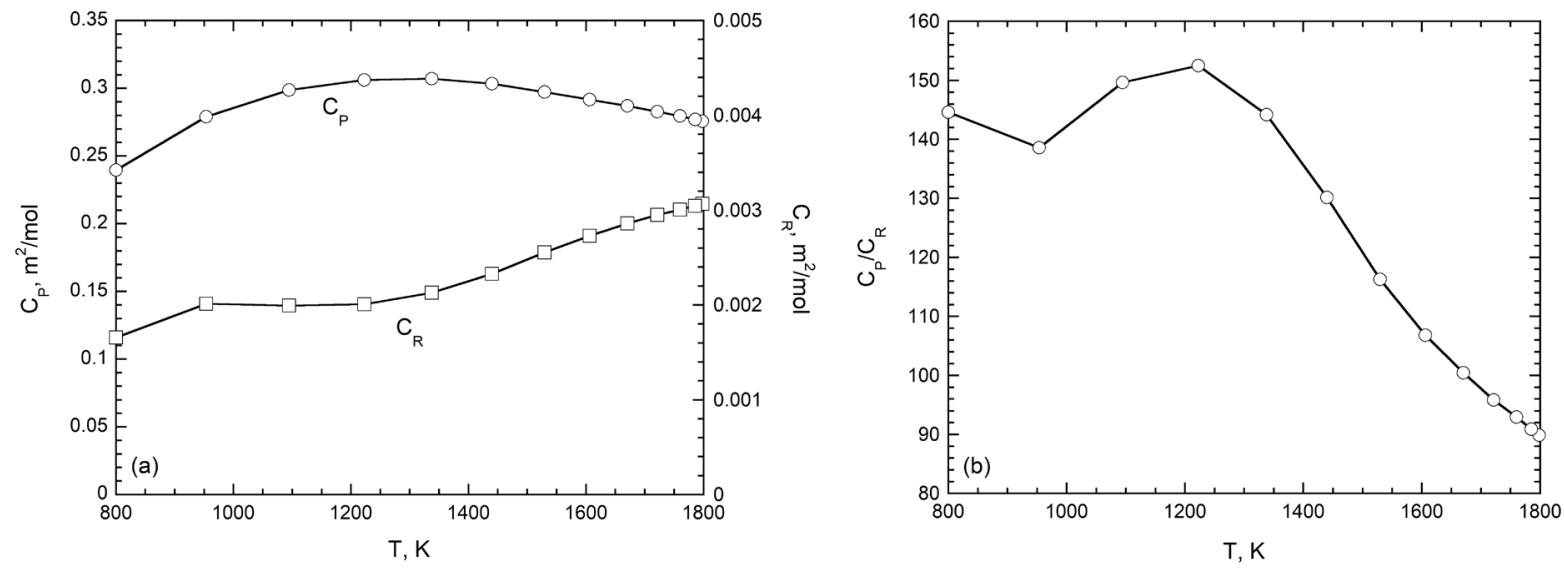

Figure 8. Dependence on temperature of the mean absorption cross-sections $C_{P}, C_{R}$, and their ratio $C_{P} / C_{R}$ for Example 1 . 
The spatial dependence of the scaling coefficients $u_{P}$ and $u_{R}$ for Example 1 is presented in Fig. 9. It may be observed that both $u_{P}$ and $u_{R}$ deviate significantly from unity, ranging from values below 0.2 to above 1.2. This suggests that considerable correction is needed from the reference state to achieve optimal results. Further, the figure reveals that the scaling coefficients vary significantly through the medium. While generalizations are not possible with a single example problem, it may be stated that the influence of the Planck mean absorption cross-section and Rosseland mean absorption cross-section on the scaling coefficient is significant.

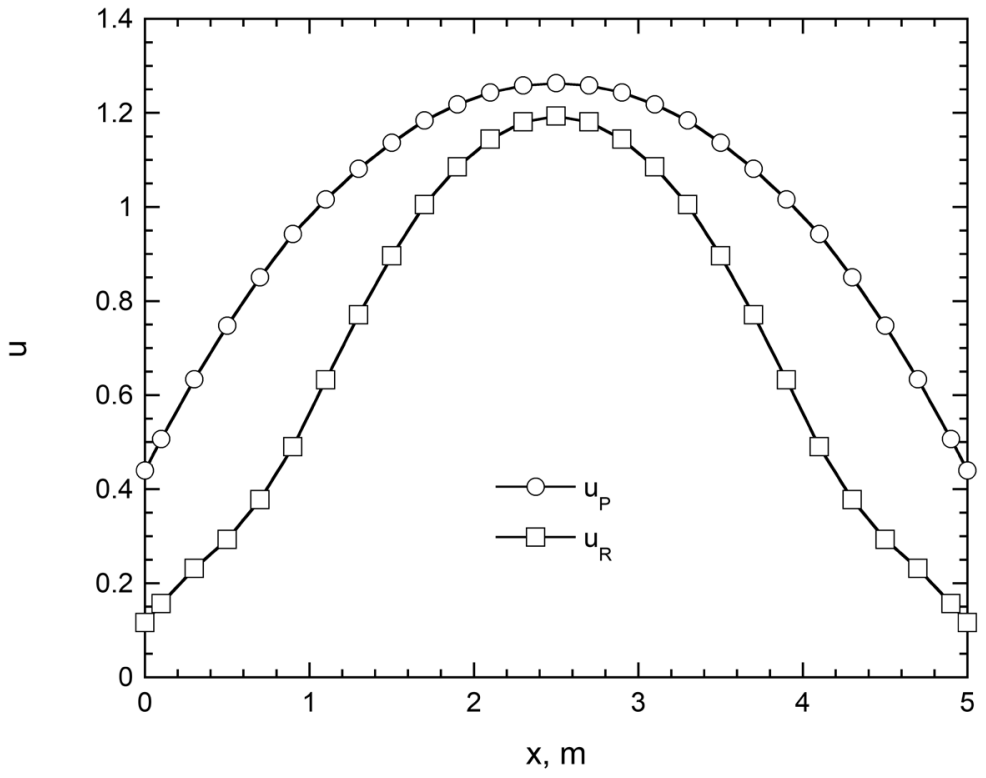

Figure 9. Profiles of the local scaling coefficients $u_{P}$ and $u_{R}$ in the gas layer of Example 1 .

Despite the simpler approach to construction of the Scaled SLW model relative to correlated SLW models, the moment-based Scaled SLW models have stronger theoretical foundation and more specific recommendations for the choice of the local scaling coefficient. The results showing more accurate prediction using the Planck mean absorption cross-section for the smaller layer thicknesses is in agreement with the Eq. (43) which is for the optically thin case. It should be noted that as $L$ increases the layer deviates from the optically thin limit. However, the scaling coefficient is established in the Multi-Layer SLW solution method [24], in which sublayers can be considered optically thin.

\section{Example 2}

Example 1 considered in the foregoing section considered a non-homogeneous mixture featuring spatially constant mole fraction ratio, $Y_{\mathrm{H}_{2} \mathrm{O}} / Y_{\mathrm{CO}_{2}}$. This situation is not atypical for gases in 
combustion applications. However, to investigate the ability of the scaled SLW approaches developed here to handle gas media with non-constant mole fraction ratio the previous example is modified in Example 2. With all other example problem parameters unchanged, the water vapor mole fraction profile is now inverted in such a way that the total water vapor in the layer remains the same:

$$
Y_{\mathrm{H}_{2} \mathrm{O}}^{i n v}=29 / 75-(4 / 5) x(x-L) / L^{2}
$$

Figure 10 illustrates the original $\mathrm{H}_{2} \mathrm{O}$ and $\mathrm{CO}_{2}$ mole fraction profiles $\left(Y_{\mathrm{H}_{2} \mathrm{O}}\right.$ and $\left.Y_{\mathrm{CO}_{2}}\right)$ used in Example 1, the inverted $\mathrm{H}_{2} \mathrm{O}$ mole fraction profile $\left(Y_{\mathrm{H}_{2} \mathrm{O}}^{i n v}\right)$ used in Example 2, and the corresponding mole fraction ratio profiles $\left(Y_{\mathrm{H}_{2} \mathrm{O}} / Y_{\mathrm{CO}_{2}}\right.$ and $\left.Y_{\mathrm{H}_{2} \mathrm{O}}^{i n v} / Y_{\mathrm{CO}_{2}}\right)$. For this problem the ratio of $\mathrm{H}_{2} \mathrm{O}$ and $\mathrm{CO}_{2}$ mole fractions varies significantly in the range $1.17<Y_{\mathrm{H}_{2} \mathrm{O}} / Y_{\mathrm{CO}_{2}}<6.4$, compared to the spatially constant ratio $Y_{\mathrm{H}_{2} \mathrm{O}} / Y_{\mathrm{CO}_{2}}=2$ for the $\mathrm{H}_{2} \mathrm{O}$ mole fraction profile of Example 1. This problem is proposed to rigorously test the Scaled SLW model's ability to perform under a condition radically departing from constant $\mathrm{H}_{2} \mathrm{O} / \mathrm{CO}_{2}$ mole fraction. It may also be noted that the problem of non-constant $\mathrm{H}_{2} \mathrm{O} / \mathrm{CO}_{2}$ mole fraction has not been explored using the Rank Correlated SLW model.

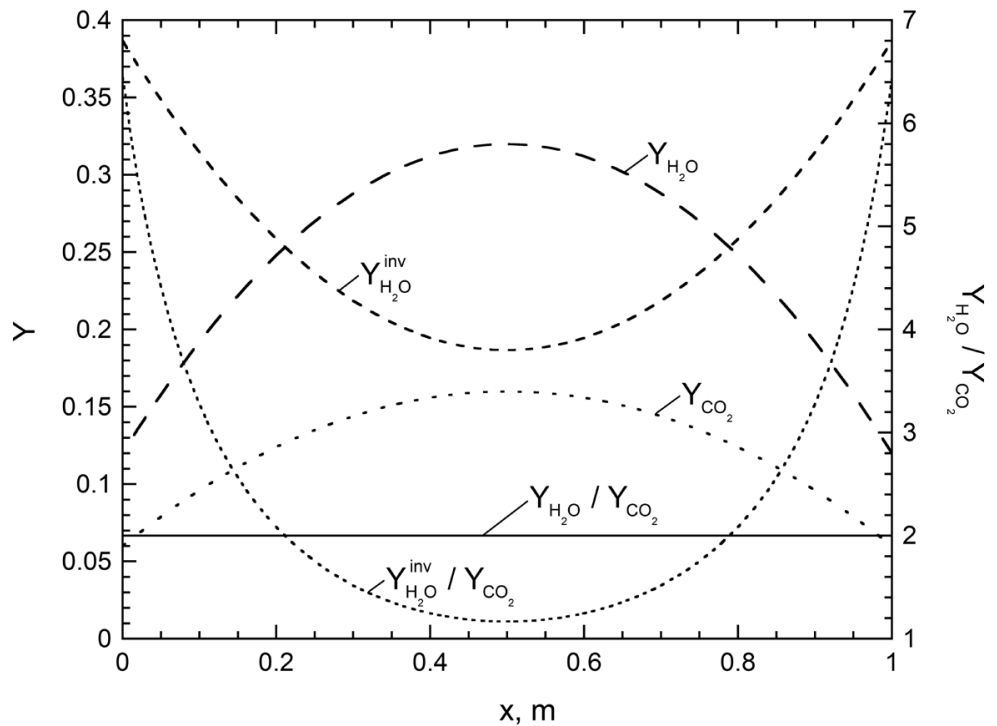

Figure 10. Mole fraction and mole fraction ratio profiles for Examples 1 and 2, illustrating the original and inverted $\mathrm{H}_{2} \mathrm{O}$ mole fraction and corresponding mole fraction ratio profiles.

Figure 11 presents predictions for the inverted $\mathrm{H}_{2} \mathrm{O}$ mole fraction profile scenario described above for a layer of thickness $L=1.0 \mathrm{~m}$. The figure includes the line-by-line benchmark solution, and the Scaled SLW model, Rank Correlated SLW model, and SLW Reference Approach model predictions. The local radiative flux divergence $Q(x)$ and local absolute relative error $L E(x)$ are shown. Consistent with the results of Example 1, there is very good agreement in 
the radiative flux divergence for the RC-SLW and RA-SLW methods using $T_{b}=T_{\text {ave }}$ in the RCSLW model and $T_{\text {ref }}=T_{\max }$ for the RA-SLW model. By contrast, it may be observed that the Scaled SLW model predictions using $T_{r e f}=T_{\max }$ yield local errors somewhat higher than for the spatially constant mole fraction ratio case of Example 1. Recall from Fig. 7 that the predicted total relative error for the constant mole fraction case of Example 1 revealed the choice of $T_{\text {ref }}=$ $T_{\max }$ to be nearly optimal for both the SP-SLW and SR-SLW models for $L=1.0 \mathrm{~m}$. Also shown in Fig. 11 are predictions using the Scaled SLW models (SP-SLW and SR-SLW) using reference temperatures $T_{r e f}=1250 \mathrm{~K}$ for the SP-SLW model and $T_{r e f}=1000 \mathrm{~K}$ for the SR-SLW model. It may be seen that the use of these reference temperatures in the predictions yields much more accurate results.

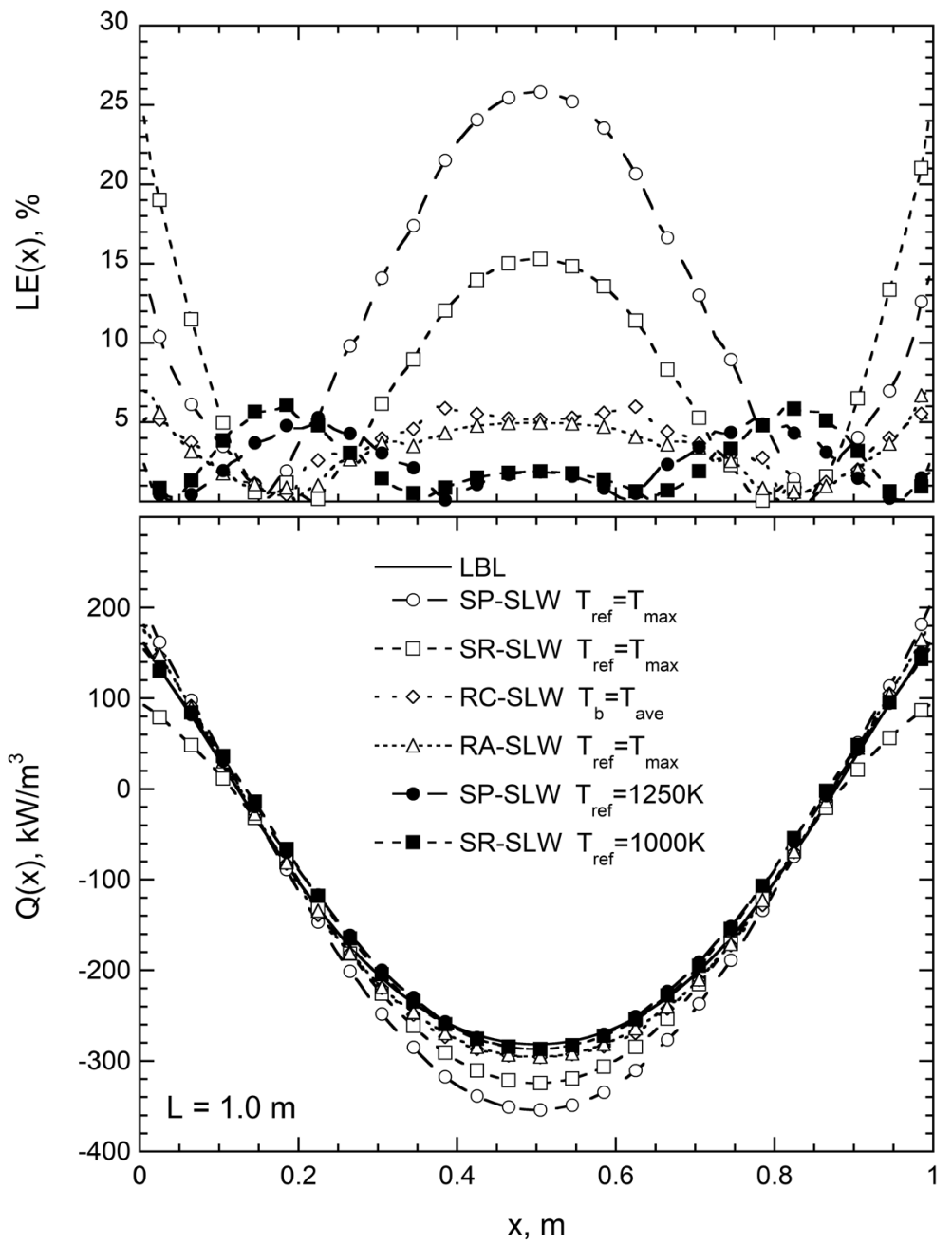

Figure 11. Predicted total divergence of the local net radiative flux $Q(x)$ and corresponding local relative error $L E(x)$ for the inverted $\mathrm{H}_{2} \mathrm{O}$ mole fraction profile of Example 2. 
The local error data of Fig. 11 suggest that for the conditions of Example 2, the optimal reference temperature deviates significantly from the maximum temperature in the domain. Figure 12 illustrates the dependence of the total absolute relative error, $T E$, on the reference temperature $T_{\text {ref }}$ (or $T_{b}$ for the RC-SLW method) for the case featuring the inverted $\mathrm{H}_{2} \mathrm{O}$ mole fraction profile, Example 2. The RA-SLW and RC-SLW methods show similar monotonic dependence on $T_{r e f}$ or $T_{b}$ to that observed in Example 1. The total error in the RA-SLW model prediction is monotonically decreasing, with the minimum total error found for $T_{r e f}=T_{\max }$. Likewise, similar to the results of Example 1, the RC-SLW model predictions are nearly insensitive to $T_{b}$, with the optimal source temperature being the minimum temperature in the domain. By contrast, the Scaled SLW model predictions show behavior quite different from that observed in Example 1. Both scaled models exhibit a local minimum in the total absolute relative error in the range of minimum and maximum temperature of the domain. As can be seen, $T E$ is a minimum near $T_{\text {ref }}$ $\approx 1250 \mathrm{~K}$ for the SP-SLW model, and $T_{r e f} \approx 1000 \mathrm{~K}$ for the SR-SLW model (for which predictions were included in the local radiative flux divergence and local error results of Fig. 11). The accuracy appears to depend quite strongly on the reference temperature for these two methods, and the use of $T_{r e f}=T_{\max }$ yields unsatisfactory results. However, the predictions shown in Fig. 12 for the non-constant mole fraction condition confirm that the Scaled Model can achieve accuracy rivaling (or even exceeding) that of the less restrictive Rank Correlated SLW model if an optimized reference temperature can be found. Further work is needed to characterize and generalize this dependence on the reference temperature.

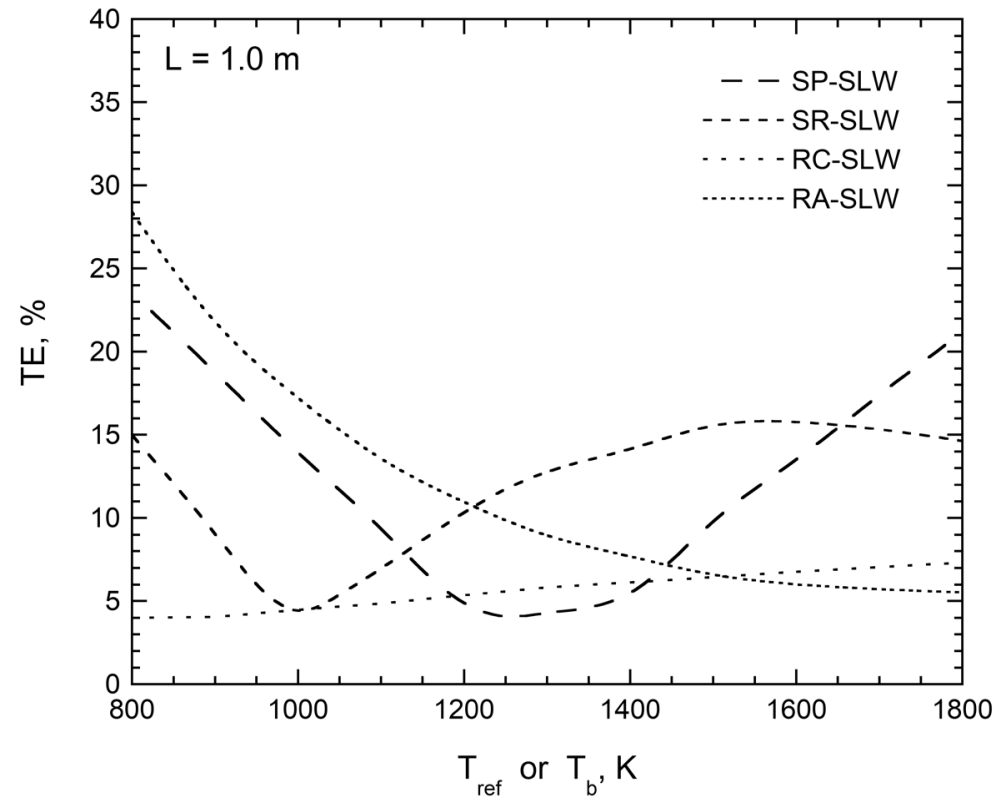

Figure 12. Predicted total relative absolute error as a function of $T_{r e f}$ for the SP-SLW, SR-SLW, RA-SLW models; and $T_{b}$ for the RC-SLW model for the inverted $\mathrm{H}_{2} \mathrm{O}$ mole fraction profile of Example 2. 


\section{OUTLINE OF THE SCALED SLW MODEL}

It may be instructive to present a step-by-step outline of the Scaled SLW model to illustrate in a detailed way its implementation.

1) One first chooses the reference gas thermodynamic state $\phi_{r e f}=\left\{T_{r e f}, Y_{r e f}, p_{r e f}\right\}$. The reference total pressure and gas species mole fractions should be chosen as spatially averaged values. For the planar layer, it is calculated by

$$
Y_{\text {ref }}=\int_{0}^{L} Y(x) d x / L
$$

Because the emission is nonlinearly weighted by temperature, in the absence of more information relative to the optimum, it is recommended to choose the maximum temperature in the medium as the reference temperature $T_{r e f}=T_{\max }$.

2) The reference supplemental absorption cross-sections are selected $\tilde{C}_{0}^{\text {ref }}, \tilde{C}_{1}^{\text {ref }}, \ldots, \tilde{C}_{n}^{\text {ref }}$ as evenly logarithmically-spaced between the minimum and the maximum values $C_{\min }$ and $C_{\max }$ which effectively represent the spectral gas absorption cross section $C_{\eta}\left(\phi_{\text {ref }}\right)$ at the reference state:

$$
\tilde{C}_{j}^{r e f}=C_{\min }\left(C_{\max } / C_{\min }\right)^{j / n}, j=0,1, \ldots, n
$$

Here, $n$ is the number of gray gases in the model ( 8 - 12 gray gases are recommended for high accuracy). Then the reference absorption cross-sections can be chosen as a geometric mean of the corresponding adjacent supplemental cross-sections

$$
C_{j}^{r e f}=\sqrt{\tilde{C}_{j-1} \tilde{C}_{j}}, j=1,2, \ldots, n
$$

Alternative subdivisions into the reference absorption cross-sections can be found in [2].

3) Calculate the reference modified Planck mean and Rosseland mean absorption cross-sections using the well-established SLW modelling approach according to Eqs. (11) and (12):

$$
\begin{aligned}
& C_{P}^{r e f}\left(\phi_{\text {ref }}, T_{b}=T_{\text {loc }}\right)=\sum_{j=1}^{n} a_{j}^{r e f} C_{j}^{r e f} \\
& \frac{1}{C_{R}^{r e f}\left(\phi_{r e f}, T_{b}=T_{l o c}\right)}=\sum_{j=1}^{n} \frac{a_{j}^{r e f}}{C_{j}^{r e f}}
\end{aligned}
$$

The local gray gas weights are calculated as 


$$
a_{j}^{r e f}=F\left(\tilde{C}_{j}^{r e f}, \phi_{r e f}, T_{b}=T_{l o c}\right)-F\left(\tilde{C}_{j-1}^{r e f}, \phi_{r e f}, T_{b}=T_{l o c}\right) .
$$

Calculate the local modified Planck mean and Rosseland mean absorption cross-sections using their SLW modelling:

$$
\begin{gathered}
C_{P}^{l o c}\left(\phi_{l o c} T_{b}=T_{l o c}\right)=\sum_{j=1}^{n} a_{j}^{l o c} C_{j}^{r e f} \\
\frac{1}{C_{R}^{l o c}\left(\phi_{l o c} T_{b}=T_{l o c}\right)}=\sum_{j=1}^{n} \frac{a_{j}^{l o c}}{C_{j}^{r e f}}
\end{gathered}
$$

where the local gray gas weights are calculated as

$$
a_{j}^{l o c}=F\left(\tilde{C}_{j}^{r e f}, \phi_{l o c}, T_{b}=T_{l o c}\right)-F\left(\tilde{C}_{j-1}^{r e f}, \phi_{l o c}, T_{b}=T_{l o c}\right) .
$$

Note that in both cases, the blackbody source temperature is chosen at the local state, $T_{b}=T_{l o c}$. The Planck mean and the Rosseland mean absorption cross-sections can be calculated also using the full data of the ALBDF look-up table [21] by Eqs. (13) and (14).

The local scaling coefficients are calculated explicitly using Eqs. (15) and (16):

$$
\begin{aligned}
& u_{P}^{l o c}=\frac{C_{P}\left(\phi_{l o c}, T_{l o c}\right)}{C_{P}\left(\phi_{r e f}, T_{l o c}\right)} \\
& u_{R}^{l o c}=\frac{C_{R}\left(\phi_{l o c}, T_{l o c}\right)}{C_{R}\left(\phi_{r e f}, T_{l o c}\right)}
\end{aligned}
$$

4) The local gray gas absorption coefficients and their weights are now calculated. The local gray gas absorption coefficients at location $s$ are defined by scaling (where $u_{P}^{l o c}$ is recommended in most cases)

$$
\kappa_{j}(s)=\kappa_{j}^{l o c}=N_{l o c} Y_{l o c} u^{l o c} C_{j}^{r e f}
$$

It is recommended that the local gray gas weights be calculated with the help of the reference supplemental cross-sections and the reference thermodynamic state (Method 1):

$$
a_{j}(s)=a_{j}^{l o c}=F\left(\tilde{C}_{j}^{r e f}, \phi_{r e f}, T_{l o c}\right)-F\left(\tilde{C}_{j-1}^{r e f}, \phi_{r e f}, T_{l o c}\right)
$$

The clear gas absorption coefficient is $\kappa_{0}(s)=\kappa_{0}^{l o c}=0$ and the corresponding weight is $a_{0}(s)=a_{0}^{\text {loc }}=F\left(\tilde{C}_{0}^{r e f}, \phi_{\text {ref }}, T_{l o c}\right)$. The gray gas weights for the boundary conditions are calculated using the wall temperature as the blackbody source temperature, $T_{b}=T_{w}$ : 


$$
\begin{aligned}
& a_{j}^{w}=F\left(\tilde{C}_{j}^{r e f}, \phi_{r e f}, T_{w}\right)-F\left(\tilde{C}_{j-1}^{r e f}, \phi_{r e f}, T_{w}\right) \\
& a_{0}^{w}=F\left(\tilde{C}_{0}^{r e f}, \phi_{r e f}, T_{w}\right) .
\end{aligned}
$$

The gray gas RTE is then written as

$$
\frac{\partial I_{j}(s)}{\partial s}=-\kappa_{j}(s) I_{j}(s)+a_{j}(s) \kappa_{j}(s) I_{b}(T(s)), j=0,1, \ldots, n
$$

Any arbitrary RTE solver can be used for solution of the gray gas RTE subject to boundary conditions.

Finally, some comment may be made relative to correlated and scaled models for the prediction of radiative transfer in nonhomogeneous gas media. Correlated models assume a perfect monotonically increasing relationship between gas spectra in distinct states. This assumption, called comonotonicity in the most recent statistical literature, is never true in practice. This can be verified by calculating Spearman's coefficient $[16,29]$, which are used for determining the level of correlation between data at different conditions. By definition, Spearman's coefficient takes a value of unity for comonotonic (completely correlated) variables and zero for data that are completely statistically independent. It can be verified that Spearman's coefficients for real gas spectra deviate from unity, and thus, gas spectra are not rigorously comonotonic. In general, real gas spectra are neither correlated nor scaled, and thus, neither approximation can be generalized to be superior. This is consistent with the findings in this work.

\section{CONCLUSIONS}

The Scaled SLW method suitable for engineering applications has been developed on the basis of the first and first inverse moments of the gas absorption cross-section. The method as developed features simple, explicit construction of the scaling coefficient, which is shown for two formulations of the scaling coefficient. Predictions reveal that the more simply constructed Scaled SLW model can yield accuracy near that of the less restrictive Rank Correlated SLW model if an optimal reference temperature can be determined. The Scaled SLW method can be an alternative to correlated SLW models when the gas absorption spectrum is not well correlated due to high non-uniformity of the medium.

\section{REFERENCES}

1. Denison MK, Webb BW. A spectral line based weighted-sum-of-gray-gases model for arbitrary RTE solvers. ASME J Heat Transfer 1993;115:1004-1012. 
2. Solovjov VP, Andre F, Lemonnier D, Webb BW. The generalized SLW model. J Physics: Conf Series 2016;676:1-36.

3. Solovjov VP, Andre F, Lemonnier D, Webb BW. The rank correlated SLW model of gas radiation in non-uniform Media. J Quant. Spectr Rad Transfer 2017;197;26-44

4. Song, TH, Viskanta R. Development of application of a spectral-group model to radiative heat transfer. ASME Paper No. 86-WA/HT-36, 1986.

5. Denison M. A spectral line-based weighted-sum-of-gray gases model for arbitrary RTE solvers. PhD dissertation, Brigham Young University, 1994, Provo, Utah, USA.

6. Denison MK, Webb BW. The spectral line-based weighted-sum-of-gray-gases model in nonisothermal nonhomogeneous media. ASME J Heat Transfer 1995;117:359-365.

7. Modest M. A weighted-sum-of-gray-gases model for arbitrary solution methods in radiative transfer. ASME J Heat Transfer 1991;113:650-656.

8. Dorigon LJ, Duciak G, Brittes R, Cassol F, Galarça M, França FHR. WSGG correlations based on HITEMP 2010 for computation of thermal radiation in non-isothermal, nonhomogeneous $\mathrm{H}_{2} \mathrm{O} / \mathrm{CO}_{2}$ mixtures. Int J Heat Mass Transfer 2013;64:863-873.

9. Kangwanpongpan T, França FHR, Silva RC, Schneider OS, Krautz HJ. New correlations for the weighted-sum-of-gray-gases model in oxy-fuel conditions based on HITEMP 2010 database. Int J Heat Mass Transfer 2012;55:7419-7433.

10. Centeno FR, Brittes R, França FHR, Ezekoye OA. Evaluation of gas radiation heat transfer in a 2D axisymmetric geometry using the line-by-line integration and WSGG models. J Quant Spectr Rad Transfer 2015;156:1-11.

11. Cassol F, Brittes R, França FHR, Ezekoye AO. Application of the weighted-sum-of-graygases model for media composed of arbitrary concentrations of $\mathrm{H}_{2} \mathrm{O}, \mathrm{CO}_{2}$ and soot. Int $\mathrm{J}$ Heat Mass Transfer 2014;79:796-806.

12. Centeno FR, Brittes R, França FHR, Silva CV. Application of the WSGG model for the calculation of gas-soot radiation in a turbulent non-premixed methane-air flame inside a cylindrical combustion chamber. Int J Heat Mass Transfer 2016;93:742-753.

13. Rothman LS, Gordon LE, Barber RJ, Dothe H, Gamache RR, Goldman A, Perevalov VI, Tashkun SA, Tennyson J. HITEMP, the high-temperature molecular spectroscopic database. J Quant Spectr Rad Transfer 2010;111:2139-2150.

14. Tencer J, Howell JR. On multilayer modeling of radiative transfer for use with the multisource $k$-distribution method for inhomogeneous media. ASME J Heat Transfer 2014;136:136;062703.

15. Zhang $\mathrm{H}$, Modest MF. Scalable multi-group full-spectrum correlated- $k$ distributions for radiative transfer calculations. ASME J Heat Transfer 2003;125:454-461.

16. Andre F, Solovjov VP, Lemonnier D, Webb BW. Co-monotonic global spectral models of gas radiation in non-uniform media based on arbitrary probability measures: Theoretical foundations. J Appl Math Modeling 2017;50:741-754.

17. Young SJ. Band model theory of radiation transport. The Aerospace Press, 2013. ISBN: 978-1-884989-25-4.

18. Weinreb MP, Neuendorfer AC. Method to apply homogeneous-path transmittance models to inhomogeneous atmospheres. J Atm Sci 1973;30:662-666. 
19. Modest MF. Narrow-band and full-spectrum $k$-distributions for radiative heat transfercorrelated- $k$ vs. scaling approximation. J Quant Spectr Rad Transfer 2003;76:69-83.

20. Modest MF, Radiative Heat Transfer, $3^{\text {rd }} E d, 2013$, Academic Press.

21. Pearson JT, Webb BW, Solovjov VP, Ma J. Efficient representation of the absorption line blackbody distribution function for $\mathrm{H}_{2} \mathrm{O}, \mathrm{CO}_{2}$, and $\mathrm{CO}$ at variable temperature, mole fraction, and total pressure. J Quant Spectr Rad Transfer 2014;138:82-96.

22. Andre $\mathrm{F}$. The $\ell$-distribution method for modeling of non-gray absorption in uniform and non-uniform gaseous medium. J Quant Spectr Rad Transfer 2016;179:19-32.

23. Andre F. An analysis of the symmetry issue in the $\ell$-distribution method of gas radiation in non-uniform gaseous media. J Quant Spectr Rad Transfer 2017;190:78-87.

24. Solovjov VP, Webb BW. Multilayer modeling of radiative transfer by SLW and CW methods in non-isothermal gaseous media. J Quant Spectr Rad Transfer 2008;109:245-257.

25. Solovjov VP, Webb BW. SLW modeling of radiative transfer in multicomponent gas mixtures. J Quant Spectr Rad Transfer 2000;65:655-672.

26. Tashkun SA, Perevalov VI. CDSD-4000: High-resolution, high temperature carbon dioxide spectroscopic databank. J Quant Spectr Rad Transfer 2011;112:1403-1410.

27. Andre F, Hou L, Roger M, Vaillon R. The multispectral gas radiation modeling: A new theoretical framework based on a multidimensional approach to $k$-distribution methods. $\mathrm{J}$ Quant Spectr Rad Transfer 2014;147:178-195.

28. Tencer J, Howell JR. A multi-source full spectrum $k$-distribution method for 1-D inhomogeneous media. J Physics: Conf Series 2012;369:01205.

29. Nelsen RB. Multivariate models and dependence concepts: Monograph on statistics and applied probability, Springer Science+Business Media, Dordrecht, 1997. 